\title{
D-Serine Signaling and NMDAR-Mediated Synaptic Plasticity Are Regulated by System A-Type of Glutamine/ D-Serine Dual Transporters
}

\author{
Oded Bodner, ${ }^{1}$ Inna Radzishevsky, ${ }^{1}$ Veronika N. Foltyn, ${ }^{1}$ Ayelet Touitou, ${ }^{1}$ Alec C. Valenta, ${ }^{2}$ Igor F. Rangel, ${ }^{3}$ \\ Rogerio Panizzutti, ${ }^{3,4}$ Robert T. Kennedy, ${ }^{2}$ Jean Marie Billard, ${ }^{5}$ and Herman Wolosker ${ }^{1}$ \\ ${ }^{1}$ Department of Biochemistry, Rappaport Faculty of Medicine, Technion-Israel Institute of Technology, Haifa 31096, Israel, ${ }^{2}$ Department of \\ Chemistry, University of Michigan, Ann Arbor, Michigan 48109, ${ }^{3}$ Institute of Biomedical Sciences, Federal University of Rio de Janeiro, RJ 21941- \\ 590, Rio de Janeiro, Brazil, ${ }^{4}$ Psychiatry Institute, Federal University of Rio de Janeiro, RJ 21941-590, Rio de Janeiro, Brazil, and ${ }^{5}$ COMETE, GIP \\ CYCERON, Normandie Université, UNICAEN, INSERM, 14000 Caen, France
}

D-serine is a physiologic coagonist of NMDA receptors (NMDARs) required for synaptic plasticity, but mechanisms that terminate D-serine signaling are unclear. In particular, the identity of unidirectional plasma membrane transporters that mediate $\mathrm{D}$-serine reuptake has remained elusive. We report that $\mathrm{D}$-serine and glutamine share the same neuronal transport system, consisting of the classic system A transporters Slc38a1 and Slc38a2. We show that these transporters are not saturated with glutamine in vivo and regulate the extracellular levels of D-serine and NMDAR activity. Glutamine increased the NMDAR-dependent long-term potentiation and the isolated NMDAR potentials at the Schaffer collateral-CA1 synapses, but without affecting basal neurotransmission in male mice. Glutamine did not increase the NMDAR potentials in slices from serine racemase knock-out mice, which are devoid of D-serine, indicating that the effect of glutamine is caused by outcompeting D-serine for a dual glutamine-D-serine transport system. Inhibition of the system A reduced the uptake of D-serine in synaptosomes and neuronal cultures of mice of either sex, while increasing the extracellular D-serine concentration in slices and in vivo by microdialysis. When compared with Slc38a2, the Slc38a1 transporter displayed more favorable kinetics toward the Denantiomer. Biochemical experiments with synaptosomes from Slc38a1 knock-down mice of either sex further support its role as a D-serine reuptake system. Our study identifies the first concentrative and electrogenic transporters mediating D-serine reuptake in vivo. In addition to their classical role in the glutamine-glutamate cycle, system A transporters regulate the synaptic turnover of D-serine and its effects on NMDAR synaptic plasticity.

Key words: D-serine; gliotransmission; glutamine; NMDA receptor; synaptic plasticity; system A

Significance Statement

Despite the plethora of roles attributed to D-serine, the regulation of its synaptic turnover is poorly understood. We identified the system A transporters Slc38a1 and Slc38a2 as the main pathway for neuronal reuptake of D-serine. These transporters are not saturated with glutamine in vivo and provide an unexpected link between the serine shuttle pathway, responsible for regulating D-serine synaptic turnover, and the glutamine-glutamate cycle. Our observations suggest that Slc38a1 and Slc38a2 have a dual role in regulating neurotransmission. In addition to their classical role as the glutamine providers, the system A transporters regulate extracellular D-serine and therefore affect NMDAR-dependent synaptic plasticity. Higher glutamine export from astrocytes would increase extracellular D-serine, providing a feedforward mechanism to increase synaptic NMDAR activation.

Received Apr. 5, 2020; revised May 22, 2020; accepted June 25, 2020.

Author contributions: 0.B., I.R., A.C.V., R.T.K., J.M.B., and H.W. designed research; 0.B., I.R., V.N.F., A.T., A.C.V., J.M.B., and H.W. performed research; I.F.R. and R.P. contributed unpublished reagents/analytic tools; $0 . B$., I.R., V.N.F., A.T., A.C.V., R.T.K., J.M.B., and H.W. analyzed data; H.W. wrote the paper.

The authors declare no competing financial interests.

This research was supported by Israel Science Foundation Grant \#337/19 (H.W.), the Allen and Jewell Prince Center for Neurodegenerative Disorders of the Brain (H.W.), the Michigan-Israel Partnership for
Research and Education funds (H.W., R.T.K.), National Institutes of Health Grant \#5R01EB003320-23 (R.T.K.), INSERM (J.M.B.), and the University of Caen Normandy (J.M.B.).

Correspondence should be addressed to Herman Wolosker at hwolosker@tx.technion.ac.il or Jean Marie Billard at jean-marie.billard@inserm.fr.

https://doi.org/10.1523/JNEUROSCI.0801-20.2020

Copyright $\odot 2020$ the authors 


\section{Introduction}

D-Serine is a gatekeeper of NMDARs that plays an essential role in synaptic plasticity (Mothet et al., 2000; Papouin et al., 2012; Le Bail et al., 2015). D-Serine is produced by the serine racemase (SR), which racemizes L-serine into D-serine in mammals and invertebrates (Wolosker et al., 1999; Dai et al., 2019). Although initially thought to be present in astrocytes, both D-serine and SR are mainly present in neurons (Kartvelishvily et al., 2006; Miya et al., 2008; Benneyworth et al., 2012; Ehmsen et al., 2013; Balu et al., 2014). Upon synthesis, D-serine is released from neurons by plasma membrane transporters like the Asc-1/Slc7a10, which mediates D-serine efflux in exchange for external amino acid substrates (Rosenberg et al., 2013; Sason et al., 2017; Neame et al., 2019). On the other hand, astrocytes synthesize the SR substrate L-serine, which shuttles to neurons to support the neuronal synthesis of D-serine by a mechanism called the serine shuttle (Wolosker and Radzishevsky, 2013; Wolosker et al., 2016; Coyle et al., 2020). Export of L-serine from astrocytes depends on the activity of the 3-phosphoglycerate dehydrogenase (Phgdh) enzyme, a process critical for the de novo synthesis of Lserine and optimal NMDAR activity (Neame et al., 2019).

The mechanisms by which $\mathrm{D}$-serine signaling terminates have been unclear. The $\mathrm{D}$-serine catabolic enzyme $\mathrm{D}$-amino acid oxidase is absent or poorly expressed in forebrain regions (Hashimoto et al., 1993; Schell et al., 1995). As a result, D-serine has a slow turnover rate in the forebrain (Dunlop and Neidle, 1997). While D-serine levels are modulated by different metabolites (Strísovský et al., 2005; Neame et al., 2019), transmitter receptors (Ma et al., 2014; Papouin et al., 2017), and SR post-translational modifications (Mustafa et al., 2007; Kolodney et al., 2016), the regulation of synaptic D-serine is poorly understood. Conceivably, removal of $\mathrm{D}$-serine from the synapse by plasma membrane transporters could terminate $\mathrm{D}$-serine signaling. However, the targeted deletion or selective inactivation of the known D-serine transporters in the brain (e.g., ASCT1/Slcla4, Asc-1/Slc7a10) does not lead to extracellular D-serine accumulation, but rather to lower D-serine levels (Sakimura et al., 2016; Sason et al., 2017; Kaplan et al., 2018). Since endogenous D-serine or glycine does not saturate the NMDARs (Chen et al., 2003a; Matsuda et al., 2010; Hammond et al., 2013), we raised the possibility that unidentified D-serine transporters modulate D-serine levels at the synapse or perisynaptic sites by mediating $\mathrm{D}$-serine reuptake to prevent unwanted NMDAR activation. We also hypothesize that acute inhibition of such D-serine reuptake systems would enhance the NMDAR activity and synaptic plasticity.

In the present study, we sought to identify unidirectional transporters that affect $\mathrm{D}$-serine signaling in vitro and in vivo. For this purpose, we conducted a low-throughput screening for amino acids that interfere with $\mathrm{D}$-serine transport. We found that glutamine inhibits the transport of both $\mathrm{D}$-serine and L-serine in vitro and in vivo by interacting with system A transporters (Slc38a1 and Slc38a2), which are capable of transporting $\mathrm{D}$-serine and are expressed by neurons. Inhibition of system A transporters mimicked the effects of glutamine in vitro and in vivo. Glutamine enhanced the isolated NMDAR potentials and the LTP at the Schaffer collateral-CA1 synapses by outcompeting $\mathrm{D}$-serine for its transport system and therefore increasing extracellular D-serine. Glutamine did not affect the isolated NMDAR potentials in slices of SR-KO mice, indicating that the effect was specific for $\mathrm{D}$-serine. Our data provide a new mechanism for modulating $\mathrm{D}$-serine signaling and keeping the local extracellular D-serine concentration below the full saturation of NMDARs. Our observations indicate that the system A transporters are not saturated in vivo, which enables the concomitant transport of D-serine, L-serine, and L-glutamine.

\section{Materials and Methods}

Materials. L-Serine and D-serine were purchased from Bachem. Acetonitrile, $\alpha$-(methylamino)isobutyric acid (MeAIB), Boc-L-cysteine, choline $\mathrm{Cl}$, deoxyribonuclease I, dithiothreitol, glutaminase, tetrahydrofuran, o-phthaldialdehyde, poly-D-lysine hydrobromide, proteinase $\mathrm{K}$, 2,3-dihydroxy-6-nitro-7-sulfonyl-benzo[f] quinoxaline (NBQX), sodium pyrophosphate, L-glutamate, L-glutamine, Percoll, and trans-4-hydroxy L-proline were obtained from Sigma-Aldrich. $\left[{ }^{14} \mathrm{C}\right] \mathrm{MeAIB}, \mathrm{D}-\left[{ }^{3} \mathrm{H}\right]$ serine, L- $\left[{ }^{3} \mathrm{H}\right]$ serine, L- $\left[{ }^{3} \mathrm{H}\right]$ glutamine, and $\mathrm{L}-\left[{ }^{3} \mathrm{H}\right]$ glutamate were purchased from PerkinElmer. Basal medium Eagle, DMEM, fetal bovine serum, L-glutamine solution, penicillin-streptomycin, trypsin-EDTA solution type $\mathrm{A}$, and soybean trypsin inhibitor were obtained from Biological Industries.

Synaptosomal preparations. Cortices of mice of either sex were dissected and homogenized at $4^{\circ} \mathrm{C}$ with $10 \mathrm{vol}$ of $0.32 \mathrm{~m}$ sucrose and $1 \mathrm{~mm}$ EDTA using a Potter-Elvehjem homogenizer with a Teflon pestle rotated at $800 \mathrm{rpm}$. Homogenates were cleared by two sequential centrifugations at $1000 \times g$ for 5 min to yield supernatant (S1). Purified synaptosomes were prepared by Percoll gradient fractionation from the $\mathrm{S} 1$ fraction as previously described (Dunkley et al., 1988). The synaptosomes were suspended in HBSS consisting of the following (mM): $137 \mathrm{NaCl}, 5.4 \mathrm{KCl}$, $0.18 \mathrm{Na}_{2} \mathrm{HPO}_{4}, 0.44 \mathrm{KH}_{2} \mathrm{PO}_{4}, 0.41 \mathrm{MgSO}_{4}, 0.49 \mathrm{MgCl}_{2}, 1.07 \mathrm{CaCl}_{2}, 5.6$ D-glucose, $4.2 \mathrm{NaHCO}_{3}, 1$ Na-pyruvate, and $10 \mathrm{HEPES}$, at pH 7.4. When the amount of tissue was limiting, the cerebral cortex or brainstem regions were manually homogenized with 18 strokes of $2 \mathrm{ml}$ glass Dounce homogenizer, and a crude synaptosomal preparation was obtained by centrifuging the $S 1$ at $12,000 \times g$ for $10 \mathrm{~min}$. The crude synaptosomal pellets were suspended in HBSS immediately before use. All animal procedures were performed in accordance with the Committee for the Supervision of Animal Experiments (Technion-Israel Institute of Technology).

Uptake of radiolabeled amino acids in synaptosomes. When indicated, crude or purified synaptosomes were incubated with $5 \mu \mathrm{M}\left[{ }^{3} \mathrm{H}\right]$-labeled $\mathrm{D}$-serine, L-serine, L-glutamine, or L-glutamate, or $50 \mu \mathrm{m}\left[{ }^{14} \mathrm{C}\right] \mathrm{MeAIB}$ in $\mathrm{HBSS}$ at $25^{\circ} \mathrm{C}$. The uptake was terminated by filtration (10-40 $\mu \mathrm{g}$ protein/sample) through $0.45 \mu \mathrm{m}$ nitrocellulose filters (Millipore) and washed three times with $9 \mathrm{ml}$ of ice-cold HBSS. Blanks were conducted by adding synaptosome reaction medium on ice. The radioactivity retained in the filters was monitored by scintillation counting. The values were normalized to the protein concentration measured using the BCA Protein Assay (Thermo Fisher Scientific). For testing the effect of 30-60 mM MeAIB, we reduced the $\mathrm{NaCl}$ concentration of the HBSS by 15-30 mM. Controls contained 15-30 mM choline chloride or 30-60 mM sucrose to preserve the osmolarity.

Primary neuronal cultures. Pregnant Sprague Dawley rats were anesthetized with isoflurane and killed by decapitation with the approval of the Committee for the Supervision of Animal Experiments (TechnionIsrael Institute of Technology). Serum-free neuronal cultures from the cerebral cortex were prepared from E16 or E17 embryos, as described previously (Rosenberg et al., 2013), with the following modifications. Briefly, embryos were harvested, and cortices were dissected in HEPES Buffered Salt Solution deprived of $\mathrm{Ca}^{2+}$ and $\mathrm{Mg}^{2+}$ (in mM: HEPES 10, pyruvate 1, glucose 5.6, $\mathrm{KH}_{2} \mathrm{PO}_{4} 0.44, \mathrm{KCl} 5.4, \mathrm{NaH}_{2} \mathrm{PO}_{4} 0.34, \mathrm{NaHCO}_{3}$ 4.2 , and $\mathrm{NaCl} 137$ ) and digested with $0.12 \%$ trypsin for $10 \mathrm{~min}$ at $37^{\circ} \mathrm{C}$. The digestion was terminated by soybean trypsin inhibitor, followed by the addition of $400 \mathrm{U}$ of DNase. Then, the tissue was mechanically dissociated with a fire-polished Pasteur pipette. After the clumps were removed, $1.5 \times 10^{5}$ cells/well were seeded in 96-well plates (Nunc) previously covered with $0.5 \mathrm{mg} / \mathrm{ml}$ poly-D-lysine. Half of the culture medium was replaced every 2-3 d and consisted of Neurobasal medium supplemented with $2 \%$ B27 and $1 \times 10^{5} \mathrm{U} / \mathrm{L}$ penicillin and $100 \mathrm{mg} / \mathrm{L}$ streptomycin. Biochemical experiments were conducted at day in vitro 4-5 (DIV4-5) and DIV10-12.

Uptake of amino acids in primary cultures. Primary neuronal cell cultures were washed three times with HBSS medium and incubated with 
HBSS supplemented with $5 \mu \mathrm{M}\left[{ }^{3} \mathrm{H}\right]$-labeled D-serine, L-serine, L-glutamine, or L-glutamate, or $50 \mu \mathrm{M}\left[{ }^{14} \mathrm{C}\right] \mathrm{MeAIB}$ in HBSS for $10-20 \mathrm{~min}$ at $25^{\circ} \mathrm{C}$. When testing high MeAIB concentrations, the concentration of $\mathrm{NaCl}$ was reduced to preserve osmolarity, and sucrose was added to the controls in place of MeAIB. Uptake was terminated by washing the cultures three times with ice-cold HBSS. Subsequently, cells were lysed by adding $100 \mu \mathrm{l}$ water, and the radioactivity was monitored by scintillation counting. The values were normalized to protein concentration measured using Bradford protein assay (Bio-Rad).

Radiolabeled amino acid release from acute cortical slices. Two- to 3month-old Sprague Dawley rats of either sex were anesthetized with isoflurane and killed in accordance with the Committee for the Supervision of Animal Experiments (Technion-Israel Institute of Technology). The cerebral cortex was cut into $400 \times 400 \mu \mathrm{m}$ slices with a McIlwain tissue chopper. The slices were washed three times with oxygenated KrebsHEPES Buffer (KB; in mM: $127 \mathrm{NaCl}, 1.3 \mathrm{NaH}_{2} \mathrm{PO}_{4}, 15$ HEPES, 10 glucose, $5 \mathrm{KCl}, 1 \mathrm{MgCl}_{2}$, and $2.5 \mathrm{CaCl}_{2}$, at $\mathrm{pH}$ 7.4). Thereafter, the slices were loaded with $5 \mu \mathrm{M} \mathrm{D}$ - $\left[{ }^{3} \mathrm{H}\right]$ serine or D- $\left[{ }^{3} \mathrm{H}\right]$ aspartate for $20 \mathrm{~min}$ in oxygenated $\mathrm{KB}$ at $37^{\circ} \mathrm{C}$. Neither $\mathrm{D}-\left[{ }^{3} \mathrm{H}\right]$ serine nor $\mathrm{D}-\left[{ }^{3} \mathrm{H}\right]$ aspartate is significantly metabolized during the period (Palmer and Reiter, 1994; Rosenberg et al., 2010). Then, the slices were washed three times with oxygenated $\mathrm{KB}$, transferred to $0.3 \mathrm{ml}$ chambers, and perfused at $0.6 \mathrm{ml} /$ min using a Suprafusion 1000 (SF-6) apparatus (Brandel) at $37^{\circ} \mathrm{C}$ for 20-25 min. Subsequently, the perfusate was collected at different times in sixtuplicates, and slices were stimulated with glutamine or drugs. The radioactivity was normalized by the total loading in the slices from each channel and expressed as a percentage of the total.

In vivo microdialysis. Ten-week-old male C57BL/6 mice were anesthetized with xylazine/ketamine and positioned in the stereotaxic instrument (lab standard with mouse adaptor, Stoelting). A CMA cannula (Harvard Apparatus) was implanted in the striatum at the coordinates $+0.8 \mathrm{~mm}$ (anterior), $\pm 1.3 \mathrm{~mm}$ (lateral), and $-2.5 \mathrm{~mm}$ (ventral), and the mice were allowed to recover for 18-24 h. Then the probe (CMA7; $6 \mathrm{kDa}$; diameter, $0.24 \mathrm{~mm}$; membrane length, $2 \mathrm{~mm}$ ) was slowly inserted and perfused for $16-18 \mathrm{~h}$ with a solution containing the following (in mM): $147 \mathrm{NaCl}, 2.7 \mathrm{KCl}, 1.2 \mathrm{CaCl}_{2}$, and $0.85 \mathrm{MgCl}_{2}$ at $0.3 \mu \mathrm{l} / \mathrm{min}$. Subsequently, samples were collected every $20 \mathrm{~min}$ for $3 \mathrm{~h}$ to verify the establishment of the baseline. Then, L-glutamine or MeAIB was administered by retrodialysis for $4 \mathrm{~h}$ using a three to four times higher drug concentration than those in slice experiments to account for the low extraction fraction of the microdialysis (typically 25\%). The average of the last four samples (last $80 \mathrm{~min}$ ) was used to calculate the effect of the drugs in comparison with the baseline. The mice were freely moving during the experiment, with access to food and water ad libitum. When measuring the effect of glutamine, the samples were treated with the glutaminase enzyme before the HPLC analysis to prevent the interference of the glutamine peak with the adjacent D-serine peak, as described previously (Radzishevsky and Wolosker, 2012). The probe position was verified by histologic analysis of the probe tract.

To quantify the extracellular glutamine concentration in the striatum, the basal glutamine was calculated from the microdialysis of 12 C57BL/6 mice, in which the values of extracellular D-serine were previously reported (Kaplan et al., 2018). We used the values of the in vivo recovery of the microdialysate in each mouse calculated from the average of the extraction fraction of the heavy isotopes of amino acids (Hershey and Kennedy, 2013), consisting of a mixture of the stable isotope-labeled D4-alanine, D3-serine, ${ }^{13} \mathrm{C}, \mathrm{D} 2$-threonine, ${ }^{15} \mathrm{~N}, \mathrm{D} 2$-glycine, and ${ }^{13} \mathrm{C} 5$-glutamic acid (Cambridge Isotope Laboratories); and D6-GABA (SigmaAldrich). The average \pm SEM extraction fraction was $0.22 \pm 0.02(n=12$ mice). The values of the extraction fraction of each isotope in this mouse cohort and the microdialysis method were reported previously (Kaplan et al., 2018).

Ex vivo electrophysiology. Experiments were conducted in accordance with European Council Directive 63/2010 regarding the care and use of animals for experimental procedures and were approved by the local ethics committee. Animals were housed under a $12 \mathrm{~h}$ light/dark cycle in a $20 \pm 2^{\circ} \mathrm{C}$ and $45 \pm 15 \%$ humidity-controlled environment with food and water available ad libitum. Transverse hippocampal slices $(400 \mu \mathrm{m})$ were obtained from 3- to 4-month-old C57BL/6 male mice. Animals were anesthetized with isoflurane before decapitation. Slices were prepared in ice-cold artificial CSF (aCSF), consisting of the following (in mм): $124 \mathrm{NaCl}, 3.5 \mathrm{KCl}$. $1.5 \mathrm{MgSO}_{4}, 2.3 \mathrm{CaCl}_{2}, 26.2 \mathrm{NaHCO}_{3}, 1.2$ $\mathrm{NaH}_{2} \mathrm{PO}_{4}$, and 11 glucose, and $\mathrm{pH} 7.4$, and placed in a holding chamber $(>100 \mathrm{ml})$ for at least $70 \mathrm{~min}$. Because we conducted additional recordings on the same day, subsequent slices were left in equilibration with aCSF for longer periods (2-6 h).

Each slice was transferred to the recording chamber and submerged continuously with aCSF pregassed with $95 \% \mathrm{O}_{2} / 5 \% \mathrm{CO}_{2}$. Extracellular recordings were obtained at $25-28^{\circ} \mathrm{C}$ from the apical dendritic layer of the CA1 area using micropipettes filled with $2 \mathrm{~m} \mathrm{NaCl}$. Presynaptic fiber volleys (PFVs) and field EPSPs (fEPSPs) were evoked by electrical stimulation of Schaffer collaterals and commissural fibers located in the stratum radiatum. For isolating the NMDAR potentials, the slices were perfused for $20 \mathrm{~min}$ with low- $\mathrm{Mg}^{2+}(0.1 \mathrm{mM})$ aCSF followed by a $15 \mathrm{~min}$ perfusion with low- $\mathrm{Mg}^{2+}(0.1 \mathrm{mM})$ aCSF supplemented with NBQX (10 $\mu \mathrm{M})$. The averaged slope of three PFVs and fEPSPs was measured using Win LTP software (Anderson and Collingridge, 2001). In control experiments, we also recorded from 3- to 4-month-old constitutive SR-KO mice (Basu et al., 2009). To evaluate the receptor activation level, the $\mathrm{fEPSP} / \mathrm{PFV}$ was monitored at increasing stimulus intensity (300, 400, and $500 \mu \mathrm{A}$ ). The effect of exogenous glutamine was assessed by determining the fEPSP/PFV ratio after perfusing for $15 \mathrm{~min}$ with aCSF containing $0.5 \mathrm{~mm}$ glutamine, then switching for aCSF containing $1 \mathrm{~mm}$ glutamine for an additional $15 \mathrm{~min}$. Thus, the slices monitored for their fEPSP/PFV ratio were washed out in aCSF for at least $135 \mathrm{~min}$ since their preparation.

When testing the effect of MeAIB (25 mM), the drug was added at least $35 \mathrm{~min}$ before recording the isolated NMDAR fEPSP/PFV ratios. The osmolarity of the aCSF was kept constant by decreasing the $\mathrm{NaCl}$ by $12.5 \mathrm{~mm}$ and adding $12.5 \mathrm{~mm}$ CholineCl (control) or $25 \mathrm{~mm}$ MeAIB.

The paired-pulse facilitation (PPF) of basal synaptic transmission was induced by electrical stimulation of afferent fibers with a paired pulse (interstimulus interval, $30 \mathrm{~ms}$ ). PPF was calculated as the ratio of the second response slope over the first one. The effect of exogenous glutamine was assessed by determining the PPF ratio $15 \mathrm{~min}$ after $0.5 \mathrm{~mm}$ glutamine was added to the aCSF. Then glutamine was increased to $1 \mathrm{~mm}$ for an additional $15 \mathrm{~min}$, and the PPF ratio was determined again.

For evoking long-term potentiation (LTP) of synaptic transmission, a test stimulus was applied every $10 \mathrm{~s}$ in the control medium and adjusted to get an fEPSP with a $0.1 \mathrm{~V} / \mathrm{s}$ baseline slope. The averaged slope of three fEPSPs was measured for $15 \mathrm{~min}$ before the delivery of a high-frequency stimulus, consisting of one train at $100 \mathrm{~Hz}$ pulses for $1 \mathrm{~s}$ at the test intensity. Testing with a single pulse was then resumed for 60 min to determine the LTP level. Thus, the LTP slices were washed out in aCSF for at least $155 \mathrm{~min}$ since their preparation. When testing the effect of L-glutamine $(1 \mathrm{mM})$, the amino acid was added $25 \mathrm{~min}$ before the establishment of the baseline and maintained throughout the recording. For better solubility, the glutamine stock solution was prepared in $0.1 \mathrm{M} \mathrm{HCl}$, giving a concentration of $1 \mathrm{~mm} \mathrm{HCl}$ in the recordings, which did not change the final $\mathrm{pH}$. For consistency, $1 \mathrm{~mm} \mathrm{HCl}$ was also added to the controls lacking glutamine.

Transport assays in transfected HEK293 cells. HEK293A cells were seeded in 96-well plates previously covered with $0.1 \mathrm{mg} / \mathrm{ml}$ poly-D-lysine and transfected with untagged mouse Slc38a1-pRK5, mouse Slc38a2pRK5, rat Slc7a10-pExpress-1 (Open Biosystems) and its ancillary subunit 4F2hc-pCMV-SPORT6 (Open Biosystems), human Slcla4-pCMV5, and rat Slc1a5-pRK5 (Rosenberg et al., 2013), or GFP-pRK5 control. Thirty-six hours after transfection, the cells were washed in HBSS and assayed for $\mathrm{D}-\left[{ }^{3} \mathrm{H}\right]$ serine or $\left[{ }^{14} \mathrm{C}\right] \mathrm{MeAIB}$ uptake as described for primary neuronal cultures. Each condition was typically assayed in octuplicates. The HEK293A cells overexpress $\mathrm{Na}^{+}$-dependent glutamine and D-serine transporters like Slcla5 (Seyer et al., 2016), which increases the value of the blanks. Unlike Slc1a5, which is an obligatory $\mathrm{Na}^{+}$-dependent transporter, the Slc38a1 and Slc38a2 transporters tolerate $\mathrm{Li}^{+}$and work better at slightly alkaline $\mathrm{pH}$ values (Reimer et al., 2000; Chaudhry et al., 2002). Therefore, to improve the signal-to-noise ratio of $\mathrm{D}-\left[{ }^{3} \mathrm{H}\right]$ serine uptake by Slc38a1 or Slc38a2, $\mathrm{Li}^{+}$was substituted for $\mathrm{Na}^{+}$in HBSS adjusted to $\mathrm{pH}$ 8.0. The uptake of $\left[{ }^{14} \mathrm{C}\right] \mathrm{MeAIB}$ by Slc38a1 or Slc38a2 
was assayed using HBSS containing $\mathrm{Na}^{+}$, at $\mathrm{pH}$ 7.4 , wherein the total $\mathrm{Na}^{+}$was reduced to balance the osmolarity of MeAIB. Blanks consisted in HEK293A cells transfected with GFP control. The uptake by all the other transporters was conducted in the presence of $\mathrm{Na}^{+}$, as described above.

Mice models. Slc38a1 ${ }^{+/-}$mice (Slc38$\mathrm{a} 1^{\text {tm1.1(KOMP)Vlcg }}$ ) were obtained from the KOMP Repository (UC Davis, Davis, CA). The mutant mice were generated by the insertion of the LacZ cassette followed by a floxed hUbCpro-neo-cassette between exons 2 and 4 (Targeting Project VG15012). After Cre-mediated removal of the neomycin cassette, the mice were then backcrossed for five generations with ICR (CD1) outbred mice and kept as + /- . Controls in uptake experiments consisted of the WT littermates. Offspring genotyping was performed with the following oligonucleotides: Reg-LacF: 5' -ACTTGCTTTAAAAAACCTCC CACA-3'; Reg-Slc38a1-wtF: 5'-GCCCGAAG ATGACAACGTCAGC-3'; Reg-Slc38a1-R: 5' GAGAAAGCAAGCACCAAGAGAGG-3'; and Reg-Slc38a1-wtR: 5'-AAATTCATATCGCATC TTCAAACAGAGC- $3^{\prime}$. The WT allele generated a 193 bp PCR fragment (primers wtF and $w t R$ ), while the $+/-$ mice exhibited an additional 594 bp fragment corresponding (primers Reg-LacF and Reg- Slc38a1-R). Constitutive Slc1a5-KO mice (TF1331, ASCT2-KO) were obtained from Taconic Biosciences, and their genotyping was determined as described previously (Kaplan et al., 2018). The mice were backcrossed in our facility with C57BL/6J mice for 10 generations. Constitutive SR-KO mice in a C57BL/6 background were obtained from Prof. Joseph T. Coyle's laboratory (Harvard Medical School, Boston, MA).

Endogenous brain amino acid levels. Levels of L- and D-amino acid enantiomers in brain regions of male Slc38a1 $1^{+/-}$and WT littermates were determined by HPLC as previously described (Hashimoto et al., 1992), except that aminoadipic acid was used as the internal standard. The GABA levels were determined by HPLC after derivatization with $o$-phtalodialdehyde and $\beta$-mercaptoethanol (Fekkes et al., 1995).

Western blots. Levels of Slc38al were determined from crude cortical synaptosomes of 6- to 10-week-old mice of either sex using 1:1000 antiSNAT1/SLC38A1 (clone D9L2P; catalog \#36057, Cell Signaling Technology) and revealed with anti-mouse 1:10,000 (Jackson ImmunoResearch). The samples from cortical synaptosomes were pretreated with the glycosidase PNGase F under denaturing conditions according to the manufacturer instructions (New England Biolabs). Samples from the brainstem were not subjected to PNGase F treatment.

LacZ detection. Adult Slc38a1 ${ }^{+/-}$C57BL/6 mice were perfused with $4 \%$ paraformaldehyde and $0.2 \%$ glutaraldehyde in $0.1 \mathrm{~m}$ phosphate buffer at $\mathrm{pH}$ 7.4. After cryoprotection with sucrose $30 \%$, the brains were cut in a freezing microtome $(20 \mu \mathrm{m}$ sections) and the sections were attached to slides. Histochemical staining for X-Gal was conducted as previously described (Watson et al., 2008). Controls consisted of WT littermates.

Experimental design and statistical tests. For analysis between two groups, we used paired or unpaired two-tailed Student's $t$ tests. When the possible outcome was anticipated to be in only one direction (see Figs. $7 B, 8 B, D, F)$, a one-tailed unpaired Student's $t$ test was used. For synaptosomes
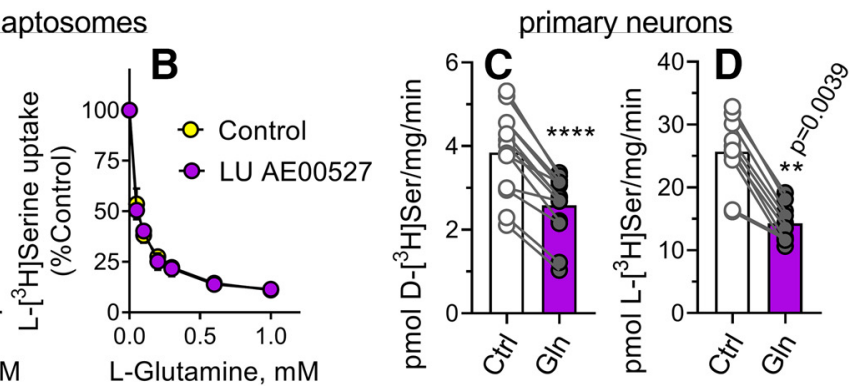

Figure 1. Glutamine affects D-serine and L-serine transport in vitro and in vivo. A, Glutamine inhibits the uptake of $5 \mu \mathrm{M}$ D- $\left[{ }^{3} \mathrm{H}\right]$ serine by purified cortical synaptosomes incubated in $\mathrm{Na}^{+}$-containing HBSS buffer and the absence $(O)$ and the presence (-) of $10 \mu \mathrm{m}$ LU AE00527, a selective Asc-1/SIC7a10 inhibitor. $\boldsymbol{B}$, Inhibition of the synaptosomal transport of L-[ $\left.{ }^{3} \mathrm{H}\right] \mathrm{ser}-$ in primary neuronal cultures. Two-tailed paired Student's $t$ test. $E$, Glutamine increases the extracellular levels of D列 0.5, or $2 \mathrm{~mm}$ glutamine when indicated. Repeated-measures ANOVA and Dunnett's multicomparison test between the last point of the baseline $(t=4.8 \mathrm{~min})$ and subsequent times. $\boldsymbol{F}-\boldsymbol{H}$, In vivo microdialysis of the striatum in adult mice. After the baseline was established, $4 \mathrm{~mm}$ glutamine was administered through retrodialysis via the probe and the levels of L-serine, Dserine, and the ratio of D-serine to total serine $(L+D)$ were determined in the microdialysate by HPLC. Ratio paired $t$ test. The values are the average \pm SEM of $3(\boldsymbol{A}), 4(\boldsymbol{B}), 13(\boldsymbol{C}), 10(\boldsymbol{D}), 4(\boldsymbol{E}$; with $0.25 \mathrm{~mm}$ and $0.5 \mathrm{~mm} \mathrm{~L}-\mathrm{Gln}), 9(\boldsymbol{E}$; with $2 \mathrm{~mm} \mathrm{~L}-$ $G \mathrm{ln})$, and $4(\boldsymbol{F}-\boldsymbol{H})$ independent experiments. Ctrl, Control.

more than two groups, the data were analyzed by repeated-measures or ordinary one-way ANOVA and Dunnett's or Sidak's multiple-comparisons test using GraphPad Prism software version 8.2.1 (GraphPad Software).

\section{Results}

A glutamine-inhibitable D-serine uptake in vitro and in vivo To identify new transport systems for D-serine, we tested the effects of several amino acids on D-serine transport. We found that glutamine inhibits the uptake of $\mathrm{D}$-serine by purified synaptosomal preparations in adult mice (Fig. $1 A$ ) with an $\mathrm{IC}_{50}$ of $214 \pm 120 \mu \mathrm{M}(n=3)$. Glutamine also inhibited the uptake of Lserine (Fig. $1 B)$ with a mean $\mathrm{IC}_{50}$ of $55 \pm 8 \mu \mathrm{M}(n=4)$. The extracellular concentration of glutamine ranges from 80 to 400 $\mu \mathrm{M}$ (Lindroth et al., 1985; Lerma et al., 1986; Kanamori and Ross, 2004; Dolgodilina et al., 2016), suggesting that the effects of 

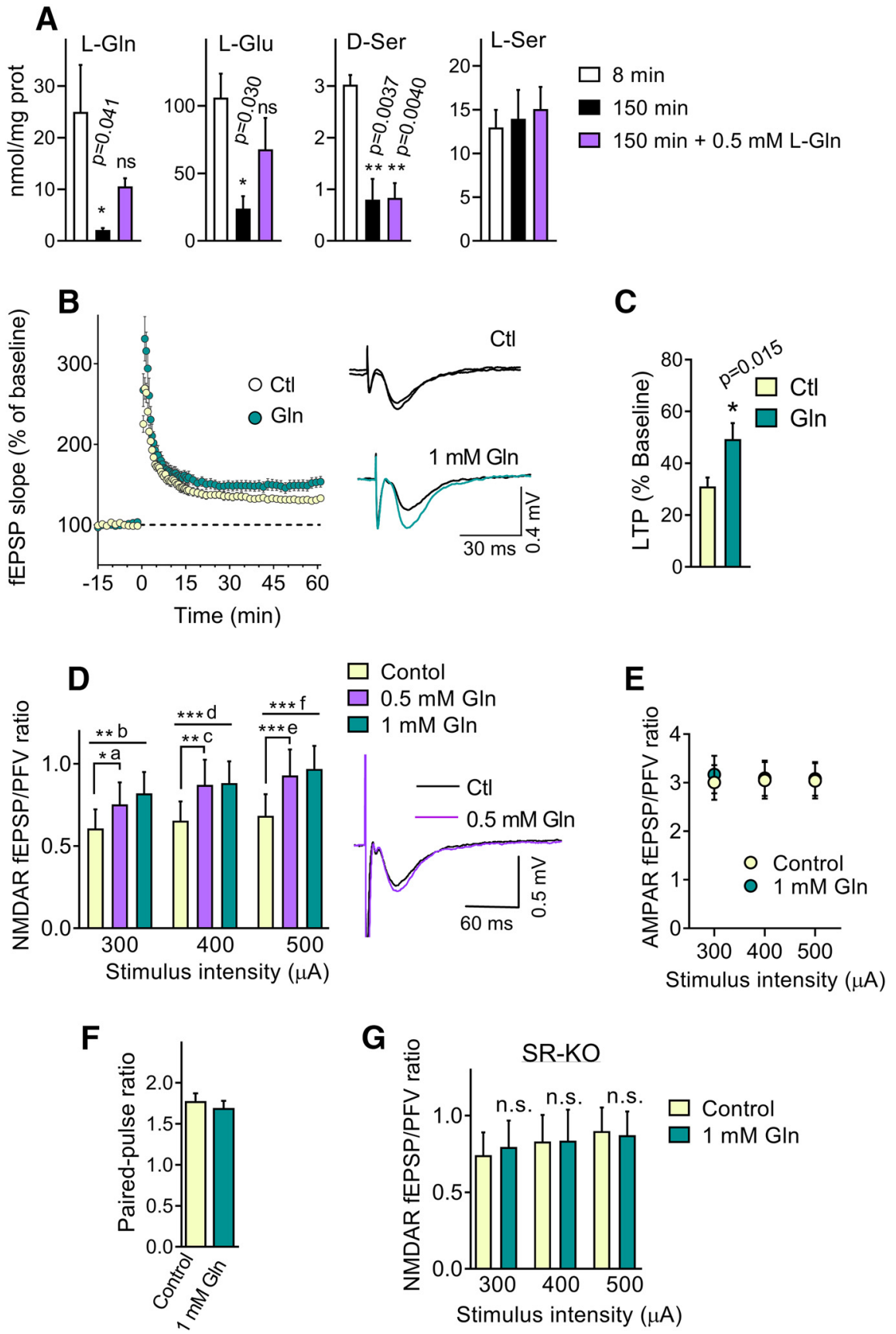

Figure 2. Glutamine stimulates the NMDAR-dependent LTP and isolated NMDAR potentials by a D-serine-dependent mechanism. $\boldsymbol{A}$, Levels of glutamine, glutamate, D-serine, and L-serine in acute slices perfused with oxygenated aCSF at $1 \mathrm{ml} / \mathrm{min}$ for 8,150 , or $150 \mathrm{~min}$ with $0.5 \mathrm{~mm}$ glutamine supplementation $(n=4)$. $\boldsymbol{B}$, LTP induced by $1 \times 100 \mathrm{~Hz}$ in the Schaffer collateralCA1 synapse of adult mice slices $(n=14$ ) was increased by $\sim 60 \%$ in slices supplemented with $1 \mathrm{~mm}$ L-glutamine added 25 min before the establishment of the baseline $(n=14)$. C, Quantification of glutamine effect on the LTP determined by the average of the last $15 \mathrm{~min}$ of recording. $\boldsymbol{D}$, The sequential addition of 0.5 and $1 \mathrm{~mm} \mathrm{L-glutamine}$ for $15 \mathrm{~min}$ to the aCSF of slices from WT mice increased the NMDAR fEPSP/PFV ratios monitored at increasing stimuli intensity $(n=16)$. $\boldsymbol{E}$, The basal synaptic transmission was not affected by $1 \mathrm{~mm}$ glutamine. The AMPA fEPSP/PFV ratio was calculated at increasing stimuli intensity $(n=19)$. $\boldsymbol{F}$, Glutamine (1 mm) did not affect the PPF of the synaptic transmission $(n=19)$, induced by stimulation of afferent fibers with paired pulses (interstimulus interval, $30 \mathrm{~ms}$ ). $\mathbf{G}$, Lack of effect of glutamine (1 mm) in the NMDAR fEPSPs of slices from SR-KO mice. Data were analyzed by one-way ANOVA and Dunnett's multiple-comparisons test $(\boldsymbol{A})$, two-tailed unpaired Student's $t$ test $(\boldsymbol{C})$, repeated-measures ANOVA and Dunnett's multiple-comparisons test $(\boldsymbol{D})$, and two-tailed paired $t$ test (G). The $p$ values are: $a, 0.037 ; b, 0.0025 ; c, 0.001 ; d, 0.0007 ; e, 0.0004 ; f,<0.0001$. Ctl, Control. n.s., not significant.

glutamine are observed in a physiological range. The inhibition by glutamine was not affected by the inclusion of the Asc-1 transporter inhibitor Lu AE000527 (Fig. 1A,B), indicating that other transport systems mediate this particular D-serine uptake in synaptosomes. We also tested glutamine in primary neuronal cortical cultures and found that it inhibits the transport of both serine enantiomers (Fig. 1C,D). To verify the effect of glutamine in a more physiological setting, we measured the extracellular D-serine levels in rat cortical slices incubated with different concentrations of glutamine. We found that perfusion with glutamine increased the extracellular concentration of D-serine (measured in the slice perfusate) at the same concentrations it inhibits D-serine uptake (Fig. 1E). To investigate the in vivo relevance of the glutamine effects, we conducted in vivo microdialysis in the striatum of adult mice. We found that retrodialysis of glutamine through the probe increased the extracellular levels of both $\mathrm{D}$-serine and L-serine (Fig. $1 F, G$ ) but without changing the ratio of D-serine to the total serine (Fig. $1 H$ ). The data indicate that glutamine likely interacts with a transport systems that uses both serine enantiomers as substrates in vitro and in vivo but without completely saturating its transport sites in vivo.

\section{A glutamine-inhibitable D-serine uptake regulates NMDAR activation}

Most of the endogenous glutamine and glutamate are lost from slices after the perfusion with aCSF (Kapetanovic et al., 1993). In agreement, we found that the perfusion of slices depletes the endogenous levels of glutamine and glutamate (Fig. $2 A)$. Interestingly, total endogenous D-serine was also moderately depleted during the perfusion, while L-serine was unaffected (Fig. 2A). Supplementation of the aCSF with $0.5 \mathrm{~mm}$ glutamine partially restored glutamine and glutamate levels in slices (Fig. 2A). However, glutamine did not increase the levels of D-serine in slices, which mostly corresponds to the intracellular D-serine pool (Fig. 2A). The data indicate that the increase in the extracellular D-serine promoted by glutamine (Fig. $1 E, F)$ is not attributable to changes in the production of $\mathrm{D}$-serine by the $\mathrm{SR}$.

In light of the inhibition of $\mathrm{D}$-serine uptake and the increase in the extracellular levels of $\mathrm{D}$-serine by glutamine application (Fig. 1), we wondered whether glutamine would increase NMDAR activation by a D-serine-dependent mechanism. In agreement, we found that adding $1 \mathrm{~mm}$ glutamine to slices for $25 \mathrm{~min}$ before starting the recordings led to a $60 \%$ increase in the extent of the NMDAR-dependent LTP at the Schaffer collateral-CA1 synapses (Fig. $2 B, C)$. Most importantly, the addition of glutamine ( 0.5 or 1 $\mathrm{mM}$ ) for $15 \mathrm{~min}$ increased the isolated NMDAR fEPSPs at different stimuli intensities (Fig. 2D). In contrast, glutamine did not 
affect the AMPAR potentials (Fig. 2E) or the paired-pulse ratio (Fig. $2 F$ ), indicating that glutamine supplementation is not required for sustaining the glutamine-glutamate cycle under our experimental conditions. This is in line with a previous report showing that exogenous glutamine is not needed to maintain the basal glutamatergic transmission or quantal size at the Schaffer collateral-CA1 synapses (Kam and Nicoll, 2007).

Since D-serine is critical for synaptic NMDAR activation at the CA1 (Le Bail et al., 2015), we investigated whether the selective augmentation of the NMDAR activity by glutamine involves an increase in the extracellular D-serine, like that seen in our biochemical experiments. To this end, we monitored the isolated NMDAR fEPSPs in slices from SR-KO mice, which display a $90 \%$ decrease in endogenous $\mathrm{D}$ serine (Basu et al., 2009). We found that glutamine $(1 \mathrm{mM})$ did not increase the isolated NMDAR fEPSPs in SR-KO mice (Fig. 2G), which suggests that an increase in the extracellular D-serine underlies the enhancement of LTP and NMDAR activation by glutamine.

\section{Characteristics of the glutamine/ \\ D-serine dual transport system}

To ascertain the identity of the glutaminesensitive D-serine transport systems, we monitored the glutamine transport in purified cortical synaptosomes of adult mice. We found that D-serine inhibits the transport of glutamine, albeit with less efficiency than L-serine (Fig. 3A). Thus, glutamine transporters may also use D-serine and L-serine as substrates, but with higher affinity to the latter. Such glutamine/Dserine dual transporter systems do not correspond to bonafide $\mathrm{D}$-serine transporters, like ASCT1 and Asc-1, which do not use glutamine as a substrate (Arriza et al., 1993; Fukasawa et al., 2000). Accordingly, inhibition of the synaptosomal uptake of both serine enantiomers by glutamine was still observed in the presence of trans-4hydroxy L-proline (OHP) or LU AE00527 (LuAE; Fig. 3B,C), which selectively inhibit ASCT1/Slcla4 (Kaplan et al., 2018) and Asc-1/Slc7a10 (Sason et al., 2017), respectively. The ASCT2/Slcla5 transporter is capable of transporting both glutamine and $\mathrm{D}$-serine in vitro (Utsunomiya-Tate et al., 1996; Rosenberg et al., 2013). In agreement with the low expression of Slcla5 in the brain (Kaplan et al., 2018), the inhibitory effect of glutamine on D-serine and L-serine uptake was also unaffected in purified synaptosomes of Slc1a5 $5^{-1-}$ mice (Fig. 3D,E).

The classical system A transporters SNAT1/Slc38al and SNAT2/Slc38a2 mediate the electrogenic uptake of glutamine in neurons (Reimer et al., 2000; Sugawara et al., 2000; Varoqui et al., 2000; Yao et al., 2000; Chaudhry et al., 2002) and account for ${ }^{* * *} p<0.001$. Ctl, Control.
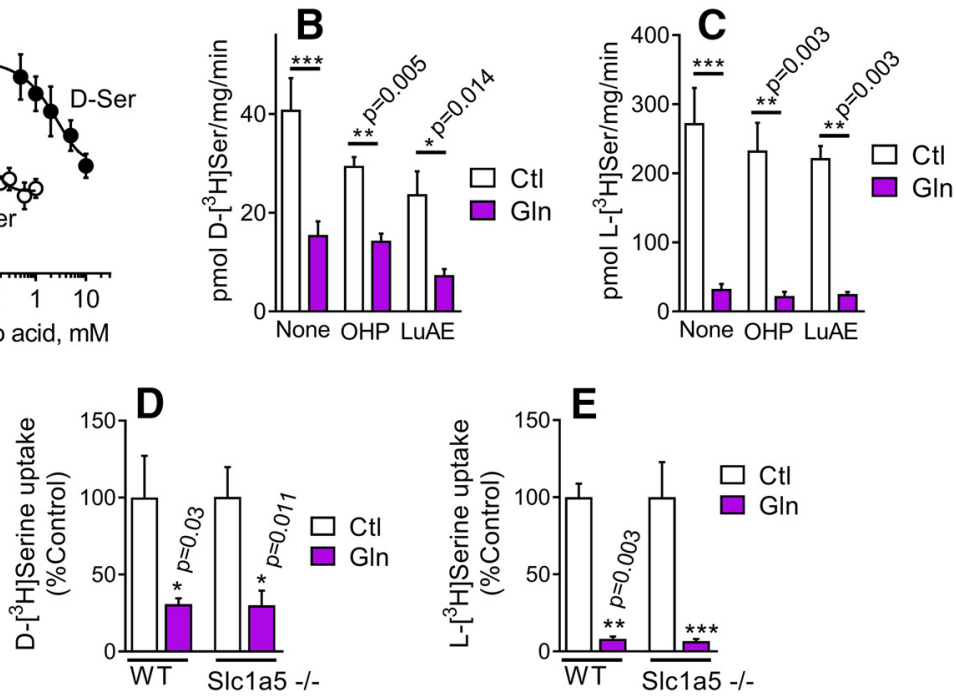

Figure 3. D-Serine is taken up through a glutamine transport system different from known D-serine transporters. A, D-serine and L-serine inhibit the uptake of $5 \mu \mathrm{m} \mathrm{L-}\left[{ }^{3} \mathrm{H}\right]$ glutamine into purified cortical synaptosomes monitored in $\mathrm{Na}^{+}$-containing HBSS. B, Glutamine (2 mM) inhibits the transport of $D-\left[^{3} H\right]$ serine in acute cortical synaptosomes, in the presence or absence of an inhibitor of Slc1a4 (1 mm OHP) or SIC7a10 (10 $\mu \mathrm{m}$ LuAE). C, Same as in $\boldsymbol{B}$ but using L-[ $\left.{ }^{3} H\right]$ serine as a substrate. D. Glutamine (2 mM) inhibited the uptake of D-[ $\left.{ }^{3} \mathrm{H}\right]$ serine in synaptosomes from Slc1a5-KO mice to the same extent as in the WT littermates. $\boldsymbol{E}$, Same as in $\boldsymbol{D}$, but with $\mathrm{L}-\left[^{3} \mathrm{H}\right]$ serine as a substrate. The values are the average \pm SEM of three to five $(\boldsymbol{A})$ and three to seven $(\boldsymbol{B}-\boldsymbol{E})$ experiments with different synaptosomal preparations. Two-tailed ratio paired $t$ test.
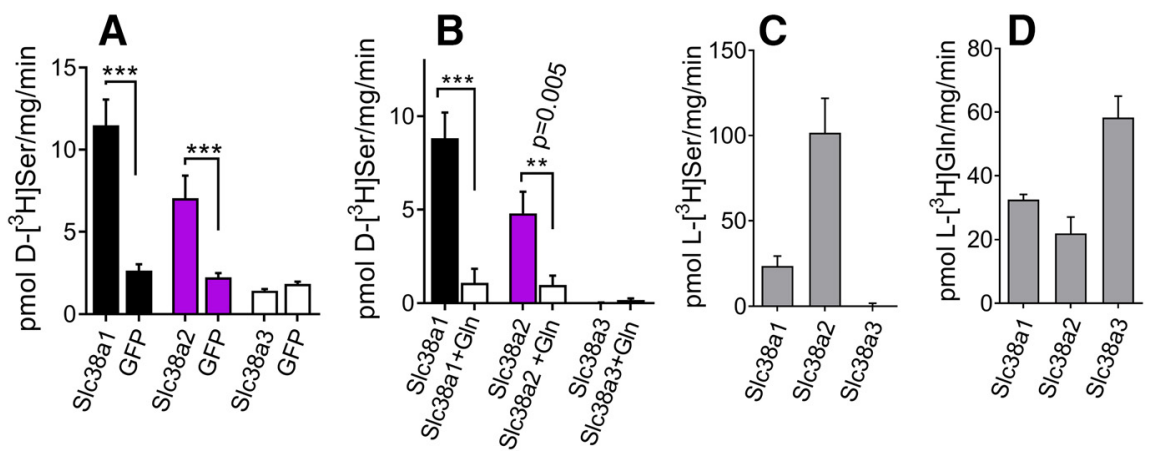

Figure 4. Uptake of D-serine by system A-type of glutamine transporters. A, Uptake of $\left.5 \mu \mathrm{m} \mathrm{D-[3}{ }^{3} \mathrm{H}\right]$ serine in HEK293A cells transfected with SIc38a1, SIc38a2, or SIc38a3 transporters compared with GFP controls monitored in HBSS containing $\mathrm{Li}^{+}$instead of $\mathrm{Na}^{+}$. $\boldsymbol{B}$, Inhibition of the D- $\left[{ }^{3} \mathrm{H}\right]$ serine uptake by $1 \mathrm{~mm} \mathrm{~L}$-glutamine in SIc38-transfected cells. C, Uptake of L-[ $\left.{ }^{3} \mathrm{H}\right]$ serine by SIc38 transporters. $\boldsymbol{D}$, Uptake of L- $\left[{ }^{3} \mathrm{H}\right]$ glutamine by SIc38 transporters. In $\boldsymbol{B}, \boldsymbol{C}$, and $\boldsymbol{D}$, the blanks were subtracted from the samples and consisted of the amino acid transport on HEK293 cells transfected with GFP control with or without glutamine addition. The values are the average \pm SEM of $18(\boldsymbol{A}$, Slc38a1), 25 ( $\boldsymbol{A}$, Slc38a2), 6 ( $\boldsymbol{A}$, Slc38a3), 4-5 (B), 3-6 (C), and 3-4 (D) different experiments. Two-tailed paired Student's $t$ test. ${ }^{* * *} p<0.0001$.

Table 1. Kinetic parameters of SIc38a1 and SIC38a2

\begin{tabular}{lllrr}
\hline & Substrate & $V_{\max }(\mathrm{pmol} / \mathrm{mg} / \mathrm{min})$ & \multicolumn{1}{c}{$K_{\mathrm{m}}(\mu \mathrm{m})$} & \multicolumn{1}{c}{$\mathrm{V}_{\max } / K_{\mathrm{m}}$} \\
\hline SIc38a1 & D-serine & $4747 \pm 770$ & $2388 \pm 595$ & $2.0 \pm 0.8$ \\
& L-serine & $5599 \pm 549$ & $846 \pm 183$ & $6.6 \pm 0.6$ \\
& L-glutamine & $7695 \pm 697$ & $667 \pm 144$ & $11.5 \pm 2.8$ \\
Slc38a2 & D-serine & $2914 \pm 432$ & $2338 \pm 555$ & $1.3 \pm 0.1$ \\
& L-serine & $17,036 \pm 1621$ & $599.7 \pm 140$ & $28.4 \pm 8.2$ \\
& L-glutamine & $6675 \pm 701$ & $838.6 \pm 194$ & $8.0 \pm 2.8$ \\
\hline
\end{tabular}

Each value corresponds to the average \pm SEM of three experiments. 

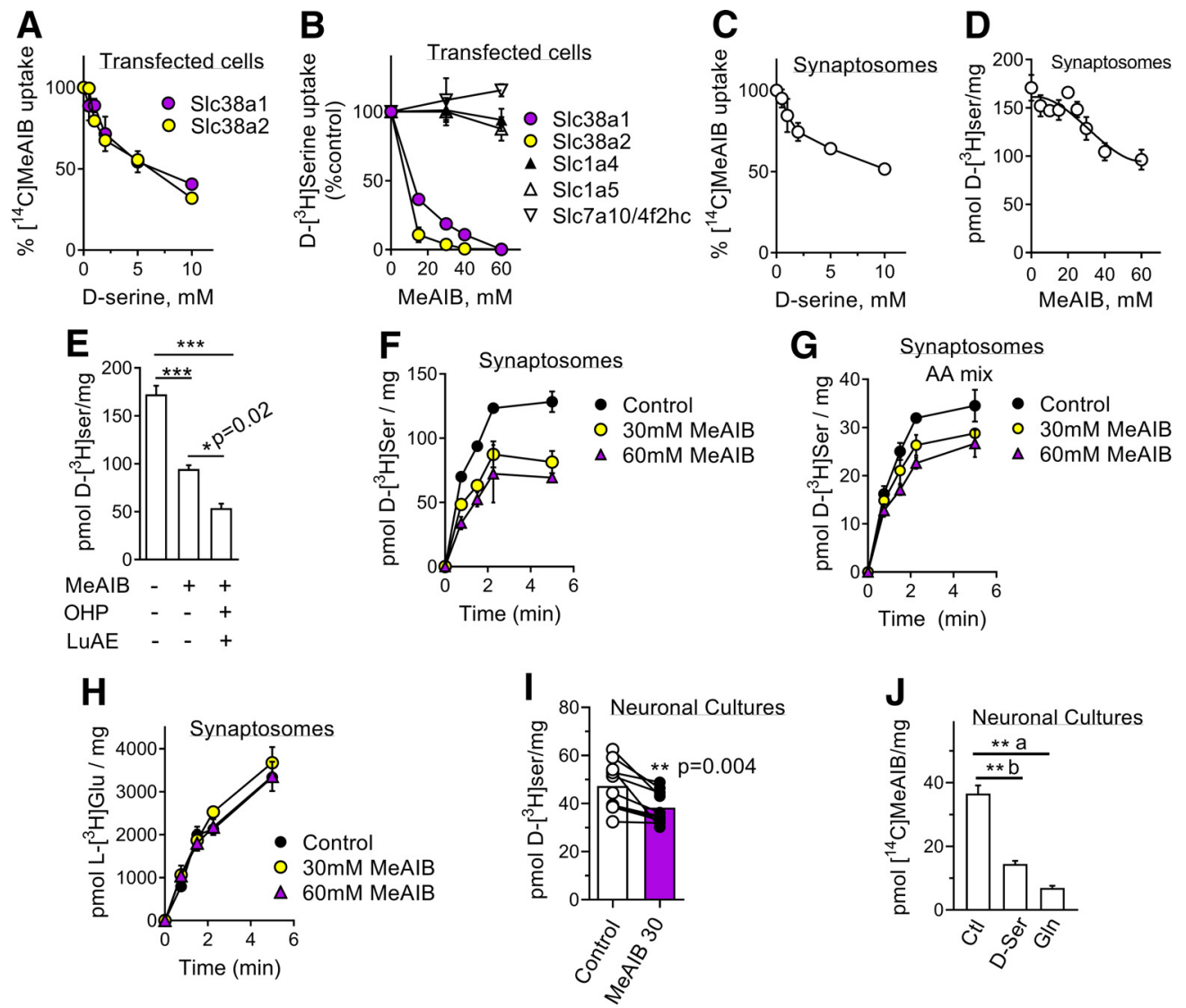

Figure 5. Mutual D-serine and MeAIB uptake by system A transporters in cells and synaptosomes. A, Uptake of $\left[{ }^{14} \mathrm{C}\right]$ MeAIB $(50 \mu \mathrm{M})$ by Slc38a1 and SIc38a2 in HEK293 transfected cells incubated in $\mathrm{Na}^{+}$-containing HBSS medium and different D-serine concentrations. Blanks consisting of cells transfected with GFP were subtracted from the values. $\boldsymbol{B}$, MeAIB inhibits the uptake of D- $\left[{ }^{3} \mathrm{H}\right]$ serine in HEK293 cells transfected with Slc38a1 and Slc38a2 but does not affect cells expressing the other D-serine transporters Slc1a4, Slc1a5, or Slc7a10/4f2hc. C, D-serine decreases the uptake of $\left[{ }^{14} \mathrm{C}\right] \mathrm{MeAIB}(50 \mu \mathrm{m})$ in purified synaptosomes of the adult mouse neocortex monitored in $\mathrm{Na}^{+}$-containing HBSS medium. $\boldsymbol{D}$, MeAlB inhibits the uptake of $5 \mu \mathrm{m} D-\left[{ }^{3} \mathrm{H}\right]$ serine in puri-

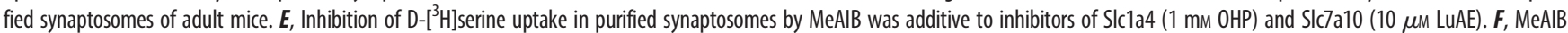
slowed down the rate of $D-\left[{ }^{3} H\right]$ serine uptake and decreased the achievable steady-state loading of synaptosomes. G, The inhibition of D-[ $\left.{ }^{3} H\right]$ serine uptake by MeAIB is still observed when the inhibitor was added together with typical system A substrates (AA mix) at physiological-like concentrations, consisting of $14 \mu \mathrm{m} \mathrm{L-alanine,} 36 \mu \mathrm{m} \mathrm{L-serine,} \mathrm{and} 230 \mu \mathrm{m} \mathrm{L-glutamine.} \boldsymbol{H}$, The uptake of L-[ $\left.{ }^{3} \mathrm{H}\right]$ glutamate $(5 \mu \mathrm{m})$ in purified synaptosomes was insensitive to MeAIB. I, MeAIB reduces the uptake of D-[3 H]serine in primary cortical neuronal cultures (DIV14). J, D-Serine (10 $\mathrm{mm})$ or glutamine $(2 \mathrm{~mm})$ inhibits the transport of $\left[{ }^{14} \mathrm{C}\right] \mathrm{MeAlB}(50 \mu \mathrm{m})$ by primary cortical neuronal cultures. All uptake experiments $(\boldsymbol{A}-\boldsymbol{J})$ were conducted in the presence of Na ${ }^{+}$. The data represent the average \pm SEM of $2(\boldsymbol{A}, \boldsymbol{H}), 3-4(\boldsymbol{B}), 4(\boldsymbol{C}, \boldsymbol{D}, \boldsymbol{E}, \boldsymbol{J}), 3(\boldsymbol{F}), 5(\boldsymbol{G})$, and $10(\boldsymbol{I})$ independent experiments. Data were analyzed by repeated-measures ANOVA and Sidak's $(\boldsymbol{E})$ or Dunnett's (I) multiple-comparison test. Two-tailed Student's paired $t$ test (I). a, $p=0.001 ; \mathrm{b}, p=0.0015{ }^{* * *} p<0.001$

$87 \%$ of the glutamine transport activity in the brain (Kanamori and Ross, 2006). In subsequent experiments, we evaluated their role as dual glutamine/D-serine transporters.

We found that Slc38a1 and Slc38a2 indeed transported D$\left[{ }^{3} \mathrm{H}\right]$ serine when transfected into HEK293 cells (Fig. 4A). In contrast, SNAT3/Slc38a3, which belongs to the system N family, did not use D-serine as a substrate (Fig. 4A). The addition of excess glutamine impaired the D-serine uptake by Slc38a1 and Slc38a2, indicating that they compete for the same transporter (Fig. 4B). As expected, transfected Slc38a1 and Slc38a2 also transported Lserine and L-glutamine (Fig. 4C,D). Since Slc38a1 and Slc38a2 are permissive to $\mathrm{Li}^{+}$(Reimer et al., 2000; Chaudhry et al., 2002; Mackenzie et al., 2003), the experiments in HEK293 cells contained $\mathrm{Li}^{+}$instead of $\mathrm{Na}^{+}$. This improved the signal by preventing the activity of other $\mathrm{Na}^{+}$-dependent $\mathrm{D}$-serine transporters endogenously expressed in HEK293 cells. Table 1 depicts the kinetic parameters of Slc38a1 and Slc38a2 toward D- $\left[{ }^{3} \mathrm{H}\right]$ serine and other substrates in transfected HEK293 cells. Slc38a2 is much more efficient for L-serine than for D-serine, while the transport rate of Slc38a1 for D-serine was closer to its L counterpart (Table 1). Under our conditions, their apparent affinities for glutamine were 0.67 and $0.82 \mathrm{~mm}$, similar to the $K_{\mathrm{m}}$ values for glutamine currents in oocytes expressing these transporters (0.52 mм; Chaudhry et al., 2002).

MeAIB is a relatively specific substrate for Slc38al and Slc38a2, albeit displaying low affinity (Chaudhry et al., 2002). DSerine inhibited the transport of $\left[{ }^{14} \mathrm{C}\right] \mathrm{MeAIB}$ by Slc38al and Slc38a2 in transfected cells monitored in the presence of sodium, confirming that $\mathrm{D}$-serine is a substrate of the system A transporters (Fig. $5 A$ ). The uptake of D- $\left[{ }^{3} \mathrm{H}\right]$ serine by Slc38a 1 and Slc38a2 in HEK293 cells was inhibited by excess MeAIB as well (Fig. $5 B$ ). Conversely, other D-serine transporters (e.g., Slc1a4, Slcla5, and Slc7a10) were insensitive to MeAIB even at very high concentrations (up to $60 \mathrm{~mm}$; Fig. 5B).

Similar to system A-overexpressing cells, D-serine inhibited the uptake of $\left[{ }^{14} \mathrm{C}\right]$ MeAIB in purified synaptosomes (Fig. $5 \mathrm{C}$ ). MeAIB also inhibited the uptake of D- $\left[{ }^{3} \mathrm{H}\right]$ serine, confirming the specificity of the effect (Fig. 5D). The inhibitory effect of MeAIB on synaptosomal D- $\left[{ }^{3} \mathrm{H}\right]$ serine uptake was additive to the Slcla4 and Slc7a10 inhibitors, consistent with the selective inactivation of system A (Fig. 5E). MeAIB slowed down the initial rate of D$\left[{ }^{3} \mathrm{H}\right]$ serine uptake and decreased the achievable steady state in 
synaptosomes (Fig. 5F), compatible with the concentrative roles of the classical system A transporters. Moreover, the inhibitory effect of MeAIB on D- $\left[{ }^{3} \mathrm{H}\right]$ serine uptake was still detectable in the presence of physiological-like extracellular concentrations of L-alanine, L- glutamine, and Lserine, indicating that other substrates do not entirely saturate the system A (Fig. 5G). The uptake of L- $\left[{ }^{3} \mathrm{H}\right]$ glutamate in synaptosomes was insensitive to MeAIB, confirming the specificity of the drug (Fig. $5 H$ ).

Similar to synaptosomes, MeAIB reduced the uptake of $\mathrm{D}-\left[{ }^{3} \mathrm{H}\right]$ serine in primary cortical neuronal cultures (Fig. 5I). As expected, excess glutamine or D-serine also diminished the transport of $\left[{ }^{14} \mathrm{C}\right] \mathrm{MeAIB}$ in primary neuronal cultures (Fig. $5 J$ ).

\section{System A inhibition alters D-serine dynamics in slices and in vivo} MeAIB increased the extracellular D- $\left[{ }^{3} \mathrm{H}\right]$ serine measured at the perfusate of acute cortical slices preloaded with $\mathrm{D}-\left[{ }^{3} \mathrm{H}\right]$ serine (Fig. 6A). The addition of D-Ile, which releases D-serine through the Slc7a10 transporter by a hetero-exchange mechanism (Rosenberg et al., 2013), caused a more substantial effect that was additive to MeAIB, indicating independent mechanisms (Fig. 6A).

Glutamine or MeAIB (5 mM) may promote small depolarization $(2-3 \mathrm{mV})$ of GABAergic interneurons of the stratum radiatum of CA1 (Chaudhry et al., 2002). However, a subsequent study found no depolarization by MeAIB in fast-spiking interneurons at the same region (Qureshi et al., 2019). To evaluate whether any small depolarization might indirectly affect amino acid release, we monitored the effect of MeAIB on D- $\left[{ }^{3} \mathrm{H}\right]$ aspartate release from slices, which is readily releasable by depolarization (Palmer and Reiter, 1994). We did not observe any $\mathrm{D}$-aspartate release by MeAIB, while depolarization with veratridine robustly releases D-aspartate (Fig. 6B).

Inhibition of system $\mathrm{A}$ transport in vivo by retrodialysis with MeAIB increased the extracellular concentrations of both D-serine (Fig. 6C) and L-serine (Fig. 6D) at the striatum, but without changing the ratio of D-serine to the total serine (Fig. $6 E$ ). In agreement with a previous study (Kanamori and Ross, 2004), MeAIB increased extracellular levels of glutamine in vivo (Fig. $6 F$ ), but did not affect those of L-glutamate (Fig. 6G). The increase in extracellular D-serine and L-serine by MeAIB suggests that the system A transporters are not saturated in vivo and also normally transport D-serine and L-serine.

There is considerable variation in the reported extracellular values of glutamine, which range from 80 to $400 \mu \mathrm{M}$ (Lindroth et al., 1985; Lerma et al., 1986; Kanamori and Ross, 2004; Dolgodilina et al., 2016). These previous studies used the low-
Slices
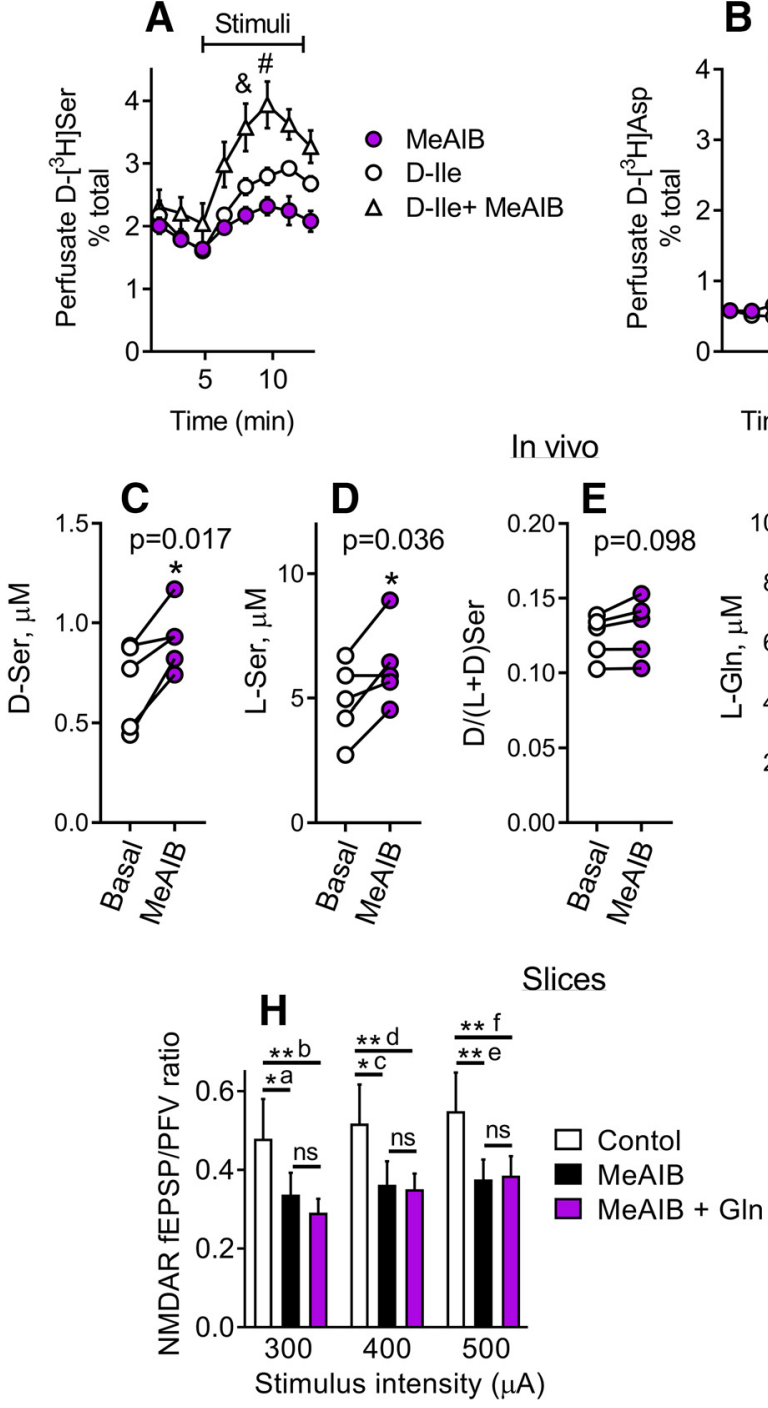

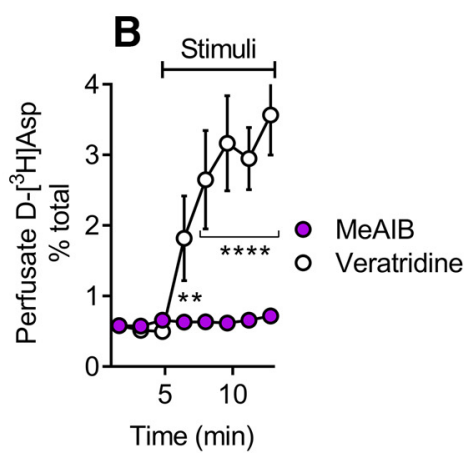

Figure 6. System A transporters regulate the extracellular levels of D-serine in vitro and in vivo, and their inhibition blocks the augmentation of the NMDAR activity by glutamine. $\boldsymbol{A}$, MeAIB $(30 \mathrm{~mm})$ increases the extracellular D-serine monitored in the perfusate of cortical slices preloaded with $\left.\mathrm{D}-{ }^{3} \mathrm{H}\right]$ serine in medium containing $\mathrm{Na}^{+}$. The increase in perfusate D-serine was additive with $1 \mathrm{~mm}$ D-isoleucine (D-lle). The osmolarity was kept constant by decreasing the amount of $\mathrm{NaCl}$ and including CholineCl in the control slices (instead of MeAIB). Different from D-lle alone; $\& p=0.046$; \#p=0.0097. $\boldsymbol{B}$, The release of D-aspartate from slices preloaded with $\mathrm{D}-\left[{ }^{3} \mathrm{H}\right]$ aspartate was not affected by MeAIB but enhanced by depolarization with $50 \mu \mathrm{m}$ veratridine. Different from MeAIB alone; ${ }^{* *} p<0.01 ;{ }^{* * *} p<0.0001$. $\mathbf{C}-\mathbf{G}$, In vivo microdialysis of the striatum of adult mice with and without MeAIB. Levels of D-serine $(\boldsymbol{C})$, L-serine $(\boldsymbol{D})$, D-serine/total serine ratio $(\boldsymbol{E})$, L-glutamine $(\boldsymbol{F})$, and L-glutamate $(\boldsymbol{G})$ in the baseline microdialysate and 2-3 h after retrodialysis with $60 \mathrm{~mm}$ MeAIB. $\boldsymbol{H}$, The addition of $25 \mathrm{~mm}$ MeAIB for 35 min decreased the isolated NMDAR fEPSPs at the Schaffer collateral-CA1 synapses of adult mice. Subsequent addition of $1 \mathrm{~mm}$ glutamine in the presence of MeAIB failed to stimulate the NMDAR fEPSPs ( $n=12$ slices). The data represent the average \pm SEM of $6(\boldsymbol{A}, \boldsymbol{B})$ or $5(\boldsymbol{C} \boldsymbol{G})$ different experiments. $\boldsymbol{A}$ and $\boldsymbol{B}$ were analyzed by two-way ANOVA and Holm-Sidak post hoc test. Data on $\boldsymbol{C}-\boldsymbol{G}$ were analyzed by two-tailed Student's $t$ test. $\boldsymbol{H}$ was analyzed by repeated-measures ANOVA, followed by Sidak's post hoc test. The $p$ values are as follows: $a, 0.047 ; b, 0.007 ; c, 0.012 ; \mathrm{d}, 0.007 ; \mathrm{e}, 0.004 ; \mathrm{f}, 0.0067$. Ctl, Control. n.s., not significant.

flow rate method to calculate the in vivo recovery of the microdialysis probe and estimate the extracellular glutamine concentrations. However, this method has many technical hurdles because of the use of very low flow rates and nonlinear regression that may lead to high variability (Chefer et al., 2009). Furthermore, Kam and Nicoll (2007) assumed the brain extracellular values for glutamine to be as high as $900 \mu \mathrm{M}$, but we could not find any reference to support this claim.

To measure the extracellular concentration of glutamine, we used retrodialysis with stable heavy isotopes of amino acids, which provides a more accurate determination of the in vivo 

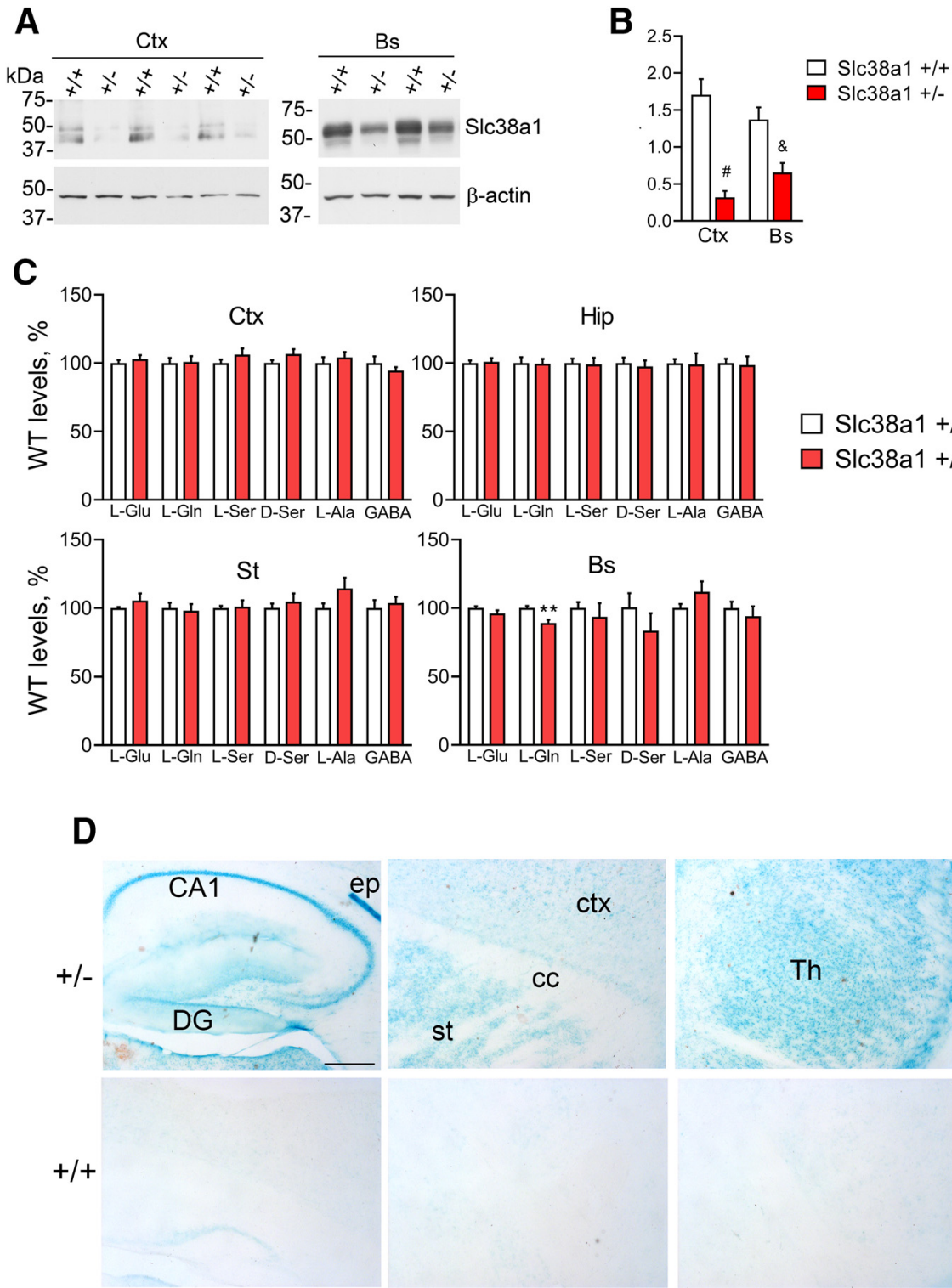

Figure 7. Effect of Slc38a1 knockdown on brain amino acid levels. $A$, Immunoblot of crude synaptosomes prepared from the neocortex (Ctx) and brainstem (Bs) of Slc38a $1^{+/-}$and WT littermates $(+/+)$. The cortical samples were treated with glycosidase as in the Materials and Methods. The bottom panel depicts the loading controls with $\beta$-actin. $\boldsymbol{B}$, Quantification of the immunoblot shows partial reduction in SIc38a1 protein in +1 - mice in both the Ctx $\left({ }^{\sharp} p=0.002, n=3\right)$ and Bs $\left({ }^{\&} p=0.007, n=4\right)$. One-tailed Student's $t$ test. C, Total amino acid levels determined by HPLC in adult SIc38a ${ }^{+/-}$mice and WT littermates (8 mice/group) show a $10 \%$ decrease in L-glutamine in the brainstem $\left({ }^{* *} p=0.0018\right.$, two-tailed Student's $t$ test), but normal levels in the other regions analyzed. Ctx, Neocortex; Hip, hippocampus; St, striatum; Bs, brainstem. $\boldsymbol{D}$, Xgal histochemistry for LacZ in Slc38a $1^{+/-}$mice (top panels) compared with the WT littermates (bottom panels). cc, Corpus callosum; DG, dentate gyrus, ep, ependymal cells; st, striatum; Th, thalamus.

recovery and neurochemical profile compared with conventional microdialysis with the low-flow rate method (Hershey and Kennedy, 2013). We calculated the extracellular glutamine concentration at the striatum from the HPLC analysis of the microdialysate from 12 C57BL/6 WT mice, previously monitored for D-serine (Kaplan et al., 2018). The extracellular glutamine calibrated for the in vivo recovery was $82.1 \pm 9.5 \mu \mathrm{M}$ (mean \pm SEM, $n=12$ mice). This value is $5-10$ times below the $K_{\mathrm{m}}$ values of system A transporters for glutamine (Table 1; Varoqui et al., 2000; Yao et al., 2000; Chaudhry et al., 2002), confirming that the classical system A transporters are not saturated in vivo.

MeAIB application in hippocampal SC-CA1 synapses transiently decreases synaptic transmission (Kam and Nicoll, 2007). In
Slc38a1 +/+

Slc38a1 +/-

the calyx of Held synapses, MeAIB decreases the amplitude of the EPSCs, suggesting that blocking glutamine uptake reduces the presynaptic glutamate levels (Billups et al., 2013). We now tested the effect of MeAIB on the glutamine-mediated enhancement of NMDAR activation. Despite the increase in extracellular D-serine promoted by MeAIB, we found that the addition of MeAIB by itself depressed the isolated NMDAR fEPSPs (Fig. 6H). Nevertheless, MeAIB prevented the augmentation of the isolated NMDAR fEPSPs by glutamine (Fig. $6 H$ ), suggesting that glutamine-mediated stimulation of NMDARs (as in Fig. 2) depends on its transport through the system A transport system.

\section{Slc38a1 knockdown affects D-serine transport}

The Slc38al has more favorable kinetics for D-serine when compared with Slc38a2 since the transport rate of Slc38a2 for L-serine surpasses $\mathrm{D}$-serine by a factor of 20 , as inferred by the maximum value $\left(\mathrm{V}_{\max }\right) / K_{\mathrm{m}}$ ratio (Table 1). Targeted deletion of Slc38al in mice depletes glutamine, GABA, and aspartate in the brain (Qureshi et al., 2019), and affects pathways involved in synaptogenesis, like mTOR (mammalian target of rapamycin; Yamada et al., 2019). Therefore, to study the role of Slc38al in D-serine transport without significantly affecting other neurotransmitters, we investigated Slc38a1 ${ }^{+/-}$ mice as a model of Slc38al knockdown. Analysis of the cerebral cortex and brainstem confirmed reduced levels of Slc38al protein expression in Slc38al ${ }^{+/-}$mice (Fig. $7 A, B)$. The total levels of Slc38al substrates in several brain regions were unchanged in Slc38 $\mathrm{al}^{+/-}$mice, except for a $10 \%$ decrease in the levels of glutamine at the brainstem (Fig. 7C), a region that exhibits a higher expression of Slc38al when compared with the cortex (Solbu et al., 2010; Yamada et al., 2019).

The Slc38a $1^{+/-}$mice have a LacZ cassette insertion that disrupts exons 2 and 4 , allowing the identification of cells that express Slc38al by using the $\beta$-galactosidase activity assay in situ, as LacZ expression is under the control of the endogenous Slc38al promoter. Using LacZ reporter as a surrogate marker for the Slc38al, we confirmed that Slc38a1 occurs in several neuronal populations, including the neurons in the pyramidal layer of the hippocampus and dentate gyrus, in the striatum and in the thalamus (Fig. $7 D$ ). In agreement with previous Slc38al staining (Mackenzie et al., 2003), strong LacZ activity was also detected in the ependyma lining the lateral ventricles (Fig. $7 D$ ). The corpus callosum was devoid of LacZ, confirming the lack of Slc38al in fibrous astrocytes (Mackenzie et al., 2003). No significant LacZ activity was detected in the WT littermates (Fig. 7D, bottom panels). 
The body weight of 14 -week-old Slc38a $1^{+/-}$mice was $\sim 10 \%$

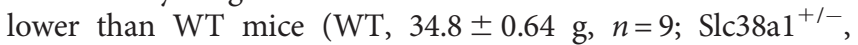
$27.7 \pm 1.1, n=16 ; p=0.021$ ). Likewise, their whole-brain weights were also $10 \%$ lower in the heterozygous mice (WT, $514 \pm$ $8.67 \mathrm{mg}, n=7$; Slc38a1 $\left.{ }^{+/-}, 472 \pm 10.64, n=7 ; p=0.0098\right)$. The lower weights imply some deleterious effect, even in our knockdown model. Therefore, we avoided behavioral or electrophysiological measurements that may be affected by pleiotropic effects and focused on characterizing the transport of D-serine and MeAIB in the Slc38 $\mathrm{al}^{+/-}$mice.

We compared the uptake of $\left[{ }^{14} \mathrm{C}\right] \mathrm{MeAIB}$ in crude synaptosomes of the cerebral cortex from Slc38a1 ${ }^{+/+}$(WT) and Slc38 $\mathrm{al}^{+/-}$(knock-down) mice with and without excess D-serine. Significantly lower uptake of $\left[{ }^{14} \mathrm{C}\right] \mathrm{MeAIB}$ was detected in preparation from Slc38al knock-down mice when compared with WT littermates (Fig. 8A). D-Serine inhibited the uptake of $\left[{ }^{14} \mathrm{C}\right] \mathrm{MeAIB}$ in WT but not significantly in the Slc38a1 ${ }^{+/-}$mice (Fig. 8A). The extent of D-serine inhibition was lower in Slc38 $\mathrm{al}^{+/-}$mice when compared with the Slc38a1 $1^{+/+}$mice (Fig. $8 B)$. Similarly, MeAIB inhibited the uptake of D- $\left[{ }^{3} \mathrm{H}\right]$ serine in cortical synaptosomes of Slc38a1 ${ }^{+/+}$mice, but had a more modest nonsignificant effect on Slc38a1 ${ }^{+/-}$mice (Fig. 8C). Analysis of the effect size shows an anticipated smaller inhibitory effect of MeAIB in synaptosomes of Slc38a1 ${ }^{+/}$mice when compared with Slc38a1 ${ }^{+/+}$mice (Fig. $8 D$ ). The data are compatible with a role of Slc38al in mediating D-serine transport in the forebrain.

Subsequently, we analyzed the D- $\left[{ }^{3} \mathrm{H}\right]$ serine uptake in crude synaptosomes from the brainstem, which expresses higher levels of Slc38a1 (Solbu et al., 2010; Yamada et al., 2019). Inhibitors of Slc7a10 and Slc1a4 were included in the assays to inactivate other $\mathrm{D}$-serine transporters. Lower uptake of $\mathrm{D}-\left[{ }^{3} \mathrm{H}\right]$ serine was observed in the brainstem synaptosomes of Slc38al knock-down mice when compared with WT littermates (Fig. 8E). The extent of MeAIB inhibition was also significantly lower in Slc38a1 ${ }^{+/-}$ mice (Fig. $8 F$ ). The data confirm that Slc38al is a physiologic regulator of D-serine metabolism by transporting $\mathrm{D}$-serine in several brain regions, providing a novel means to regulate NMDAR-dependent synaptic plasticity.

\section{Discussion}

Our study uncovered an unexpected link between the serine shuttle pathway, responsible for regulating D-serine synaptic turnover, and the glutamine-glutamate cycle (Fig. 9). We found that the system A transporters Slc38a1 and Slc38a2 are not saturated in vivo and use both serine enantiomers and glutamine as substrates. The evidence of a shared D-serine and glutamine transport system comes from our experiments using synaptosomes, primary neuronal cultures, electrophysiology, and in vivo microdialysis.

The connection of glutamine to the metabolism of D-serine was uncovered by experiments demonstrating that supplementation of the aCSF with $0.5-1 \mathrm{~mm}$ glutamine increased the NMDAR-dependent LTP and isolated NMDAR potentials in hippocampal slices. Glutamine did not affect the NMDAR fEPSPs in slices from SR-KO mice, which lack D-serine, suggesting that changes in extracellular D-serine mediate the electrophysiological effects of glutamine. In control experiments, we found that supplementation of slices with $1 \mathrm{~mm}$ glutamine did not affect the basal neurotransmission via AMPARs or the paired-pulse ratio, suggesting that glutamine did not change the glutamate release. In agreement, a similar application of glutamine did not affect the quantal amplitude of the miniature
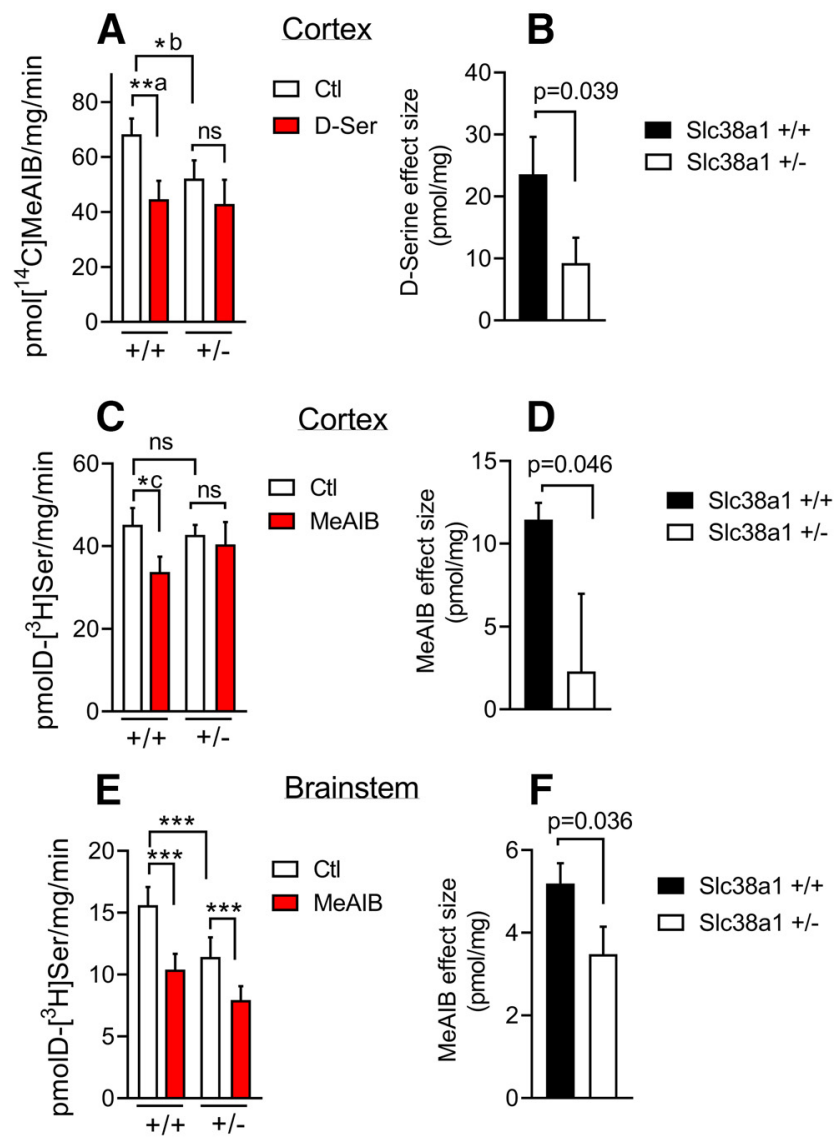

Figure 8. Lower transport of MeAIB and D-serine in Slc38a1 knock-down mice. $A$, Uptake of $\left[{ }^{14} \mathrm{C}\right] \mathrm{MeAIB}(50 \mu \mathrm{m})$ and inhibition by $2 \mathrm{~mm}$ D-serine in crude synaptosomes prepared from the cerebral cortex of Slc38a $1^{+/+}$and Slc38a $1^{+/-}$mice $(n=6)$. a, $p=0.0016 ; b, p=0.028$. B, The extent of inhibition of $\left[{ }^{14} C\right]$ MeAIB uptake by D-serine was larger in $+/+$ mice when compared with $+/-$ mice. C, Uptake of D- $\left.{ }^{3} \mathrm{H}\right]$ serine $(5 \mu \mathrm{m})$ and inhibition by $60 \mathrm{~mm}$ MeAlB in cortical crude synaptosomes prepared from Slc38a $1^{+/+}$and Slc38a $1^{+/-}$mice $(n=6) . C$, $p=0.049$. D, The extent of inhibition of D- $\left[{ }^{3} H\right]$ serine uptake by MeAlB was larger in $+/+$ mice when compared with $+/-$ mice. $E$, D- $\left[{ }^{3} H\right]$ serine $(5 \mu \mathrm{m})$ uptake and inhibition by $30 \mathrm{~mm}$ MeAIB in crude synaptosomes prepared from the brainstem of Slc38a ${ }^{+/+}$and Slc38a ${ }^{+/-}$ mice $(n=5)$. The uptake medium contained $10 \mu \mathrm{m} \mathrm{LU}$ AE00527 and $1 \mathrm{~mm}$ OHP to inhibit other D-serine transporters. ${ }^{* * *} p<0.001$. $\boldsymbol{D}$, The extent of inhibition of $\left.\mathrm{D}-{ }^{3} \mathrm{H}\right]$ serine uptake by MeAIB was larger in Slc38a $1^{+/+}$mice. All data represent the average and SEM. Data on $A, C$, and $\boldsymbol{E}$ were analyzed by repeated-measures ANOVA and Sidak's multiple-comparison test. $\boldsymbol{B}, \boldsymbol{D}$, and $\boldsymbol{F}$ were analyzed by one-tailed unpaired Student's $\boldsymbol{t}$ test. n.S., not significant.

EPSCs in the Schaffer collateral-CA1 synapses (Kam and Nicoll, 2007), indicating that the hippocampal slices contain enough endogenous glutamine to sustain basal glutamatergic neurotransmission. Our data suggest that changes in D-serine underlie the enhancement of the NMDAR fEPSPs by glutamine. Since excess exogenous glutamine or MeAIB inhibited the uptake of D-serine and increased extracellular D-serine levels in slices and in vivo, the enhancement of NMDAR activity by the glutamine is attributable to the competition with D-serine for the system A.

Previous studies assumed that system A transporters are normally saturated with glutamine (Fricke et al., 2007; Brown and Mathews, 2010; Tani et al., 2010). In contrast, our study indicates that the system A transporters are not saturated under physiologic conditions and can transport $\mathrm{D}$-serine along with glutamine for the following reasons: (1) our biochemical and electrophysiological studies with WT and SR-KO mice as controls show that glutamine increases NMDAR activity in slices by increasing extracellular D-serine; (2) D-serine uptake in synaptosomes was sensitive to MeAIB inhibition even in the presence of 


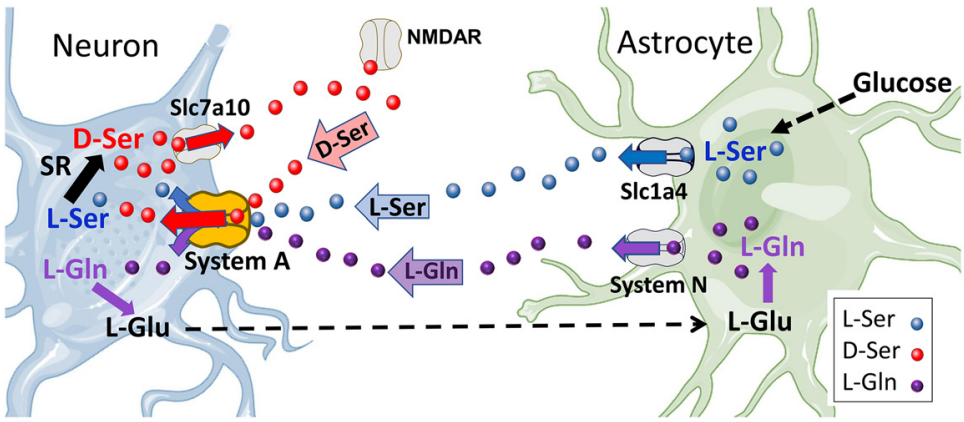

Figure 9. Proposed role of the classical system A transporters in the serine shuttle. The synaptic turnover of D-serine depends on the serine shuttle. The SR substrate L-serine is synthesized from glucose through the Phgdh pathway in astrocytes (Wolosker and Radzishevsky, 2013; Neame et al., 2019). L-Serine is subsequently exported from astrocytes by the Slc1a4 transporter and is required for the D-serine synthesis by the neuronal SR (Kaplan et al., 2018). Release of the neuronal D-serine by SIc7a10 or other pathways activate synaptic NMDARs (Rosenberg et al., 2010, 2013; Sason et al., 2017). The system A transporters (SIc38a1 and SIc38a2) mediate D-serine reuptake along with the transport of glutamine (via the glutamine-glutamate cycle) into different types of neurons. L-Serine is also a substrate of system A. This working model predicts that higher export of glutamine from astrocytes through system $\mathrm{N}$ transporters will affect D-serine reuptake mechanisms and NMDAR activation. Templates for the figure were adapted from Servier Medical Art (https://smart.servier.com)

physiological extracellular concentrations of typical system A substrates (glutamine, alanine, L-serine); (3) increasing extracellular glutamine or adding MeAIB by retrodialysis increased the extracellular levels of D-serine in vivo; and (4) when adjusted for the in vivo recovery using retrodialysis with stable isotopes, our microdialysis revealed that the extracellular glutamine concentration in freely moving mice is about one order of magnitude below the values required for half saturating the system $\mathrm{A}$ transporters.

On the other hand, we do not discard the idea that dynamic increases in glutamine at some synapses might locally inhibit the D-serine transport, providing another layer of regulation of NMDARs. For instance, the glutamine concentration at the glutamatergic calyx of Held synapses may saturate the system A transporters when astrocytes are stimulated (Uwechue et al., 2012). Selective activation of the glutamate transport by astrocytes juxtaposed to these synapses releases glial glutamine within milliseconds, evoking system A-dependent currents similar to those elicited by millimolar glutamine concentrations (Uwechue et al., 2012). The exact mechanism underlying such rapid astrocytic release of glutamine remains to be clarified. Furthermore, it is also possible that excessive synaptic activity such as that induced with high-frequency stimulation of glutamatergic afferents in the hippocampus alters glutamine availability enough to modify extracellular D-serine concentration.

The transport of D-serine by system A may be altered in pathology. A massive increase in the levels of extracellular glutamine takes place during hyperammonemia, reaching values as high as $6.5 \mathrm{~mm}$ in patients (Tofteng et al., 2006). Such high levels of extracellular glutamine will prevent the uptake of $\mathrm{D}$-serine by system $\mathrm{A}$ and contribute to NMDAR activation. In agreement, hyperammonemic rats exhibit a substantial increase in extracellular glutamine, and this is associated with NMDAR overactivation as well (Kanamori and Ross, 2005; Cauli et al., 2007).

Hatanaka et al. (2002) previously detected a slight transport of D-serine by Slc38a1 and Slc38a2 in human retinal pigment epithelial cells, but the results were not considered to be of physiological relevance. Overall, our observations indicate that Slc38a1 and Slc38a2 account for 30-50\% of D-serine transport in synaptosomes and are significant players in $\mathrm{D}$-serine uptake in vivo. The efficiency of Slc38a2 for L-serine uptake surpasses D- serine by 20 -fold, while the transport rate of Slc38a1 for L-serine is only three times that of $\mathrm{D}$-serine, as measured by the $\mathrm{V}_{\max } /$ $K_{\mathrm{m}}$ ratio. Our observations support the notion that Slc38a1 is more permissive to Denantiomers and is in agreement with a previous study reporting that $\mathrm{D}$-alanine induces larger currents in Slc38a1-expressing oocytes when compared with Slc38a2 (Mackenzie et al., 2003).

Most importantly, we found the synaptosomal uptake of D-serine to be less inhibited by MeAIB in Slc38a1 knockdown mice. Inhibition of the MeAIB uptake by $\mathrm{D}$-serine was also blunted in cortical synaptosomes of Slc38al knockdown mice. D-Serine uptake by brainstem synaptosomes was lower in Slc38al knockdown mice as well, confirming the role of Slc38a1 in mediating D-serine uptake.

The relative roles of Scl38a1 and Slc38a2 in mediating $\mathrm{D}$-serine uptake will likely depend on the synapse type. GABAergic interneurons of the hippocampus express high levels of Slc38a1 (Chaudhry et al., 2002; Mackenzie et al., 2003; Melone et al., 2004; Solbu et al., 2010), but are mostly devoid of Slc38a2 (González-González et al., 2005). Hippocampal interneurons display robust staining for D-serine despite their relatively low levels of SR when compared with glutamatergic neurons (Miya et al., 2008; Balu et al., 2014). Therefore, high Slc38al expression may account for the high levels of D-serine in GABAergic interneurons of the hippocampus.

In glutamatergic neurons, Slc38a2 is likely to be at least as important as Slc38a1 in mediating D-serine reuptake. Slc38a2 expression is particularly high in the dendrites of pyramidal cell glutamatergic neurons in the neocortex and CA1 region (Yao et al., 2000; González-González et al., 2005). These cells have the highest content of SR and D-serine, which are concentrated in dendrites and spines, consistent with an autocrine role of D-serine (Kartvelishvily et al., 2006; Miya et al., 2008; Benneyworth et al., 2012; Ehmsen et al., 2013; Lin et al., 2016; Neame et al., 2019; Coyle et al., 2020). Although Slc38al is enriched in GABAergic interneurons, this transporter is also expressed in glutamatergic pyramidal cells of the CA1 region (Mackenzie et al., 2003; Melone et al., 2004), and we detected substantial LacZ reporter activity in the pyramidal cell layer of Slc38a1 ${ }^{+/-}$mice.

D-Serine is preferentially synthesized in neurons through the serine shuttle (Wolosker and Radzishevsky, 2013; Neame et al., 2019). This metabolic pathway encompasses the export of L-serine from astrocytes to sustain the neuronal synthesis of D-serine (Fig. 9). L-Serine does not bind to the NMDAR coagonist site. However, its availability is essential for the synthesis of D-serine by SR, and therefore is critical for the NMDAR-dependent synaptic plasticity (Neame et al., 2019). However, the identity of the $\mathrm{L}$-serine import systems in neurons remained elusive. We found that glutamine and MeAIB inhibit L-serine transport and increase extracellular L-serine in vivo, implicating Slc38a1 and Slc38a 2 as potential mediators of the import of L-serine in neurons (Fig. 9). Slc38a2 seems to be a particularly attractive candidate for this role because of its much higher efficiency to L-serine when compared with D-serine (Table 1).

Despite their mechanistic role in mediating L-serine uptake, the system A transporters do not appear to be essential for providing the $\mathrm{L}$-serine required for $\mathrm{D}$-serine production under our 
experimental conditions. If this were the case, then adding excess glutamine to inhibit the uptake of L-serine would reduce D-serine synthesis and impair the NMDAR activity by diminishing the D-serine pool. However, glutamine stimulated the NMDAR activity and LTP, indicating that the buildup of extracellular Dserine promoted by glutamine (Fig. 1) prevails in the short term. Furthermore, glutamine did not reduce the total levels of D-serine in slices (Fig. 2A), suggesting that disrupting the L-serine uptake through system A did not diminish the intracellular Dserine pool under our conditions. It is also possible that other neutral amino acid transporters that use L-serine as substrate (e.g., Slc7a10, Slc7a5, or Slc7a8) compensate for system A inhibition. Alternatively, since D-serine displays low turnover $(4 \% / \mathrm{h}$ in vivo; Dunlop and Neidle, 1997), it may take several hours to deplete its intracellular pool following the inhibition of L-serine uptake. Therefore, our data do not discard that long-lasting inhibition of the system A-dependent L-serine uptake will affect NMDAR activity at a different time scale.

A few other transporters are known to use glutamine, D-serine, or MeAIB as substrates, but none of them fit with the characteristics of the D-serine transport we observed in our study. The Slcla5 transporter uses both glutamine and D-serine as substrates, but not MeAIB; its role was also excluded by our D-serine uptake experiments using synaptosomes of Slcla5 $5^{-1-}$ mice. PAT1/Slc36a1 uses both MeAIB and D-serine as substrates (Chen et al., 2003b), but it does not recognize glutamine (Sagné et al., 2001; Wreden et al., 2003). PAT2/Slc36a2 recognizes Dserine but is less efficient with L-serine or glutamine (Boll et al., 2002), which is the opposite of the kinetics we observed. Slc36a1 and Slc36a2 are restricted to lysosomes, though the latter is also present at the endoplasmic reticulum and recycling endosomes of neurons (Rubio-Aliaga et al., 2004). SNAT8/Slc38a8 transports MeAIB in oocytes, but it does not use L-serine as a substrate (Hägglund et al., 2015). Likewise, the glutamine transporter SNAT7/Slc38a7 does not use either L-serine or MeAIB (Hägglund et al., 2011). Erickson recently reported a highaffinity uptake of MeAIB $\left(K_{\mathrm{m}}=30 \mu \mathrm{M}\right)$ by an unknown glutamine transporter that is strongly stimulated by depolarization (Erickson, 2017). However, we did not observe a high-affinity inhibitory component of MeAIB on D-serine transport, excluding the role of this new glutamine transporter in D-serine dynamics. It is possible, however, that other unknown glutamine transporters also contribute to the uptake of $\mathrm{D}$-serine in addition to the classical system A transporters.

In summary, our study identified the first concentrative and electrogenic transporters capable of mediating D-serine uptake. Our observations suggest that Slc38a1 and Slc38a2 have a dual role in regulating neurotransmission. In addition to their classical role as the glutamine providers, especially under increased synaptic activity (Tani et al., 2010; Billups et al., 2013), the system A transporters regulate extracellular D-serine and therefore affect NMDAR-dependent synaptic plasticity. Higher glutamine export from astrocytes by the glutamate-glutamine shuttle would slowdown $\mathrm{D}$-serine reuptake and increase extracellular $\mathrm{D}$-serine, providing a feedforward mechanism to increase synaptic NMDAR activation.

\section{References}

Anderson WW, Collingridge GL (2001) The LTP Program: a data acquisition program for on-line analysis of long-term potentiation and other synaptic events. J Neurosci Methods 108:71-83.

Arriza JL, Kavanaugh MP, Fairman WA, Wu YN, Murdoch GH, North RA, Amara SG (1993) Cloning and expression of a human neutral amino acid transporter with structural similarity to the glutamate transporter gene family. J Biol Chem 268:15329-15332.

Balu DT, Takagi S, Puhl MD, Benneyworth MA, Coyle JT (2014) D-serine and serine racemase are localized to neurons in the adult mouse and human forebrain. Cell Mol Neurobiol 34:419-435.

Basu AC, Tsai GE, Ma CL, Ehmsen JT, Mustafa AK, Han L, Jiang ZI, Benneyworth MA, Froimowitz MP, Lange N, Snyder SH, Bergeron R, Coyle JT (2009) Targeted disruption of serine racemase affects glutamatergic neurotransmission and behavior. Mol Psychiatry 14:719-727.

Benneyworth MA, Li Y, Basu AC, Bolshakov VY, Coyle JT (2012) Cell selective conditional null mutations of serine racemase demonstrate a predominate localization in cortical glutamatergic neurons. Cell Mol Neurobiol 32:613-624.

Billups D, Marx MC, Mela I, Billups B (2013) Inducible presynaptic glutamine transport supports glutamatergic transmission at the calyx of Held synapse. J Neurosci 33:17429-17434.

Boll M, Foltz M, Rubio-Aliaga I, Kottra G, Daniel H (2002) Functional characterization of two novel mammalian electrogenic proton-dependent amino acid cotransporters. J Biol Chem 277:22966-22973.

Brown MN, Mathews GC (2010) Activity- and age-dependent modulation of GABAergic neurotransmission by system A-mediated glutamine uptake. J Neurochem 114:909-920.

Cauli O, López-Larrubia P, Rodrigues TB, Cerdán S, Felipo V (2007) Magnetic resonance analysis of the effects of acute ammonia intoxication on rat brain. Role of NMDA receptors. J Neurochem 103:1334-1343.

Chaudhry FA, Schmitz D, Reimer RJ, Larsson P, Gray AT, Nicoll R, Kavanaugh M, Edwards RH (2002) Glutamine uptake by neurons: interaction of protons with system A transporters. J Neurosci 22:62-72.

Chefer VI, Thompson AC, Zapata A, Shippenberg TS (2009) Overview of brain microdialysis. Curr Protoc Neurosci 47:7.1.1-7.1.28.

Chen L, Muhlhauser M, Yang CR (2003a) Glycine tranporter-1 blockade potentiates NMDA-mediated responses in rat prefrontal cortical neurons in vitro and in vivo. J Neurophysiol 89:691-703.

Chen Z, Fei YJ, Anderson CM, Wake KA, Miyauchi S, Huang W, Thwaites DT, Ganapathy V (2003b) Structure, function and immunolocalization of a proton-coupled amino acid transporter (hPAT1) in the human intestinal cell line Caco-2. J Physiol 546:349-361.

Coyle JT, Balu D, Wolosker H (2020) D-Serine, the shape-shifting NMDA receptor co-agonist. Neurochem Res 45:1344-1353.

Dai X, Zhou E, Yang W, Zhang X, Zhang W, Rao Y (2019) D-Serine made by serine racemase in Drosophila intestine plays a physiological role in sleep. Nat Commun 10:1986.

Dolgodilina E, Imobersteg S, Laczko E, Welt T, Verrey F, Makrides V (2016) Brain interstitial fluid glutamine homeostasis is controlled by blood-brain barrier SLC7A5/LAT1 amino acid transporter. J Cereb Blood Flow Metab 36:1929-1941.

Dunkley PR, Heath JW, Harrison SM, Jarvie PE, Glenfield PJ, Rostas JA (1988) A rapid Percoll gradient procedure for isolation of synaptosomes directly from an S1 fraction: homogeneity and morphology of subcellular fractions. Brain Res 441:59-71.

Dunlop DS, Neidle A (1997) The origin and turnover of D-serine in brain. Biochem Biophys Res Commun 235:26-30.

Ehmsen JT, Ma TM, Sason H, Rosenberg D, Ogo T, Furuya S, Snyder SH, Wolosker H (2013) D-serine in glia and neurons derives from 3-phosphoglycerate dehydrogenase. J Neurosci 33:12464-12469.

Erickson JD (2017) Functional identification of activity-regulated, high-affinity glutamine transport in hippocampal neurons inhibited by riluzole. J Neurochem 142:29-40.

Fekkes D, van Dalen A, Edelman M, Voskuilen A (1995) Validation of the determination of amino acids in plasma by high-performance liquid chromatography using automated pre-column derivatization with ophthaldialdehyde. J Chromatogr B Biomed Appl 669:177-186.

Fricke MN, Jones-Davis DM, Mathews GC (2007) Glutamine uptake by system A transporters maintains neurotransmitter GABA synthesis and inhibitory synaptic transmission. J Neurochem 102:1895-1904.

Fukasawa Y, Segawa H, Kim JY, Chairoungdua A, Kim DK, Matsuo H, Cha $\mathrm{SH}$, Endou H, Kanai Y (2000) Identification and characterization of a $\mathrm{Na}$ $(+)$-independent neutral amino acid transporter that associates with the $4 \mathrm{~F} 2$ heavy chain and exhibits substrate selectivity for small neutral Dand L-amino acids. J Biol Chem 275:9690-9698. 
González-González IM, Cubelos B, Giménez C, Zafra F (2005) Immunohistochemical localization of the amino acid transporter SNAT2 in the rat brain. Neuroscience 130:61-73.

Hägglund MG, Sreedharan S, Nilsson VC, Shaik JH, Almkvist IM, Bäcklin S, Wrange O, Fredriksson R (2011) Identification of SLC38A7 (SNAT7) protein as a glutamine transporter expressed in neurons. J Biol Chem 286:20500-20511.

Hägglund MGA, Hellsten SV, Bagchi S, Philippot G, Löfqvist E, Nilsson VCO, Almkvist I, Karlsson E, Sreedharan S, Tafreshiha A, Fredriksson R (2015) Transport of L-glutamine, L-alanine, L-arginine and L-histidine by the neuron-specific Slc38a8 (SNAT8) in CNS. J Mol Biol 427:14951512.

Hammond S, Seymour CM, Burger A, Wagner JJ (2013) D-Serine facilitates the effectiveness of extinction to reduce drug-primed reinstatement of cocaine-induced conditioned place preference. Neuropharmacology 64:464-471.

Hashimoto A, Nishikawa T, Oka T, Takahashi K, Hayashi T (1992) Determination of free amino acid enantiomers in rat brain and serum by high-performance liquid chromatography after derivatization with $\mathrm{N}$ tert.-butyloxycarbonyl-L-cysteine and o-phthaldialdehyde. J Chromatogr 582:41-48.

Hashimoto A, Nishikawa T, Konno R, Niwa A, Yasumura Y, Oka T, Takahashi K (1993) Free D-serine, D-aspartate and D-alanine in central nervous system and serum in mutant mice lacking D-amino acid oxidase. Neurosci Lett 152:33-36.

Hatanaka T, Huang W, Nakanishi T, Bridges CC, Smith SB, Prasad PD, Ganapathy ME, Ganapathy V (2002) Transport of D-serine via the amino acid transporter ATB $(0,+)$ expressed in the colon. Biochem Biophys Res Commun 291:291-295.

Hershey ND, Kennedy RT (2013) In vivo calibration of microdialysis using infusion of stable-isotope labeled neurotransmitters. ACS Chem Neurosci 4:729-736.

Kam K, Nicoll R (2007) Excitatory synaptic transmission persists independently of the glutamate-glutamine cycle. J Neurosci 27:9192-9200.

Kanamori K, Ross BD (2004) Quantitative determination of extracellular glutamine concentration in rat brain, and its elevation in vivo by system $\mathrm{A}$ transport inhibitor, alpha-(methylamino)isobutyrate. J Neurochem 90:203-210.

Kanamori K, Ross BD (2005) Suppression of glial glutamine release to the extracellular fluid studied in vivo by NMR and microdialysis in hyperammonemic rat brain. J Neurochem 94:74-85.

Kanamori K, Ross BD (2006) Kinetics of glial glutamine efflux and the mechanism of neuronal uptake studied in vivo in mildly hyperammonemic rat brain. J Neurochem 99:1103-1113.

Kapetanovic IM, Yonekawa WD, Kupferberg HJ (1993) Time-related loss of glutamine from hippocampal slices and concomitant changes in neurotransmitter amino acids. J Neurochem 61:865-872.

Kaplan E, Zubedat S, Radzishevsky I, Valenta AC, Rechnitz O, Sason H, Sajrawi C, Bodner O, Konno K, Esaki K, Derdikman D, Yoshikawa T, Watanabe M, Kennedy RT, Billard JM, Avital A, Wolosker H (2018) ASCT1 (Slc1a4) transporter is a physiologic regulator of brain D-serine and neurodevelopment. Proc Natl Acad Sci U S A 115:9628-9633.

Kartvelishvily E, Shleper M, Balan L, Dumin E, Wolosker H (2006) Neuronderived $\mathrm{D}$-serine release provides a novel means to activate $\mathrm{N}$-methyl-Daspartate receptors. J Biol Chem 281:14151-14162.

Kolodney G, Dumin E, Safory H, Rosenberg D, Mori H, Radzishevsky I, Wolosker H (2016) Nuclear compartmentalization of serine racemase regulates D-serine production. IMPLICATIONS FOR N-METHYL-dASPARTATE (NMDA) RECEPTOR ACTIVATION. J Biol Chem 291:2630.

Le Bail M, Martineau M, Sacchi S, Yatsenko N, Radzishevsky I, Conrod S, Ait Ouares K, Wolosker H, Pollegioni L, Billard JM, Mothet JP (2015) Identity of the NMDA receptor coagonist is synapse specific and developmentally regulated in the hippocampus. Proc Natl Acad Sci U S A 112: E204-E213.

Lerma J, Herranz AS, Herreras O, Abraira V, Martín del Río R (1986) In vivo determination of extracellular concentration of amino acids in the rat hippocampus. A method based on brain dialysis and computerized analysis. Brain Res 384:145-155.

Lin H, Jacobi AA, Anderson SA, Lynch DR (2016) D-serine and serine racemase are associated with PSD-95 and glutamatergic synapse stability. Front Cell Neurosci 10:34
Lindroth P, Hamberger A, Sandberger M (1985) Liquid chromatography determination of amino acids after precolumn fluorescent derivatization. In: Amino acids (neuromethods), Ed 1 (Boulton AA, Baker GB, Wood JD, eds), pp 97-116. Clifton, NJ: Humana.

Ma TM, Paul BD, Fu C, Hu S, Zhu H, Blackshaw S, Wolosker H, Snyder SH (2014) Serine racemase regulated by binding to stargazin and PSD-95: potential N-methyl-D-aspartate- $\alpha$-amino-3-hydroxy-5-methyl-4-isoxazolepropionic acid (NMDA-AMPA) glutamate neurotransmission crosstalk. J Biol Chem 289:29631-29641.

Mackenzie B, Schäfer MK, Erickson JD, Hediger MA, Weihe E, Varoqui H (2003) Functional properties and cellular distribution of the system A glutamine transporter SNAT1 support specialized roles in central neurons. J Biol Chem 278:23720-23730.

Matsuda S, Matsuzawa D, Nakazawa K, Sutoh C, Ohtsuka H, Ishii D, Tomizawa H, Iyo M, Shimizu E (2010) D-serine enhances extinction of auditory cued fear conditioning via ERK1/2 phosphorylation in mice. Prog Neuropsychopharmacol Biol Psychiatry 34:895-902.

Melone M, Quagliano F, Barbaresi P, Varoqui H, Erickson JD, Conti F (2004) Localization of the glutamine transporter SNAT1 in rat cerebral cortex and neighboring structures, with a note on its localization in human cortex. Cereb Cortex 14:562-574.

Miya K, Inoue R, Takata Y, Abe M, Natsume R, Sakimura K, Hongou K, Miyawaki T, Mori H (2008) Serine racemase is predominantly localized in neurons in mouse brain. J Comp Neurol 510:641-654.

Mothet JP, Parent AT, Wolosker H, Brady RO Jr, Linden DJ, Ferris CD, Rogawski MA, Snyder SH (2000) D-serine is an endogenous ligand for the glycine site of the N-methyl-D- aspartate receptor. Proc Natl Acad Sci U S A 97:4926-4931.

Mustafa AK, Kumar M, Selvakumar B, Ho GP, Ehmsen JT, Barrow RK, Amzel LM, Snyder SH (2007) Nitric oxide S-nitrosylates serine racemase, mediating feedback inhibition of D-serine formation. Proc Natl Acad Sci U S A 104:2950-2955.

Neame S, Safory H, Radzishevsky I, Touitou A, Marchesani F, Marchetti M, Kellner S, Berlin S, Foltyn VN, Engelender S, Billard JM, Wolosker H (2019) The NMDA receptor activation by D-serine and glycine is controlled by an astrocytic Phgdh-dependent serine shuttle. Proc Natl Acad Sci U S A 116:20736-20742.

Palmer AM, Reiter CT (1994) Comparison of the superfused efflux of preaccumulated $\mathrm{D}$-[3H]aspartate and endogenous L-aspartate and L-glutamate from rat cerebrocortical minislices. Neurochem Int 25:441-450.

Papouin T, Ladépêche L, Ruel J, Sacchi S, Labasque M, Hanini M, Groc L, Pollegioni L, Mothet JP, Oliet SH (2012) Synaptic and extrasynaptic NMDA receptors are gated by different endogenous coagonists. Cell 150:633-646.

Papouin T, Dunphy JM, Tolman M, Dineley KT, Haydon PG (2017) Septal cholinergic neuromodulation tunes the astrocyte-dependent gating of hippocampal NMDA receptors to wakefulness. Neuron 94:840-854.e7.

Qureshi T, Sørensen C, Berghuis P, Jensen V, Dobszay MB, Farkas T, Dalen KT, Guo C, Hassel B, Utheim TP, Hvalby O, Hafting T, Harkany T, Fyhn M, Chaudhry FA (2019) The glutamine transporter Slc38a1 regulates GABAergic neurotransmission and synaptic plasticity. Cereb Cortex 29:5166-5179.

Radzishevsky I, Wolosker H (2012) An enzymatic-HPLC assay to monitor endogenous D-serine release from neuronal cultures. Methods Mol Biol 794:291-297.

Reimer RJ, Chaudhry FA, Gray AT, Edwards RH (2000) Amino acid transport system A resembles system $\mathrm{N}$ in sequence but differs in mechanism. Proc Natl Acad Sci U S A 97:7715-7720.

Rosenberg D, Kartvelishvily E, Shleper M, Klinker CM, Bowser MT, Wolosker H (2010) Neuronal release of D-serine: a physiological pathway controlling extracellular D-serine concentration. FASEB J 24:2951-2961.

Rosenberg D, Artoul S, Segal AC, Kolodney G, Radzishevsky I, Dikopoltsev E, Foltyn VN, Inoue R, Mori H, Billard JM, Wolosker H (2013) Neuronal D-serine and glycine release via the Asc-1 transporter regulates NMDA receptor-dependent synaptic activity. J Neurosci 33:3533-3544.

Rubio-Aliaga I, Boll M, Vogt Weisenhorn DM, Foltz M, Kottra G, Daniel H (2004) The proton/amino acid cotransporter PAT2 is expressed in neurons with a different subcellular localization than its paralog PAT1. J Biol Chem 279:2754-2760

Sagné C, Agulhon C, Ravassard P, Darmon M, Hamon M, El Mestikawy S, Gasnier B, Giros B (2001) Identification and characterization of a 
lysosomal transporter for small neutral amino acids. Proc Natl Acad Sci U S A 98:7206-7211.

Sakimura K, Nakao K, Yoshikawa M, Suzuki M, Kimura H (2016) A novel $\mathrm{Na}(+)$-independent alanine-serine-cysteine transporter 1 inhibitor inhibits both influx and efflux of D-serine. J Neurosci Res 94:888-895.

Sason H, Billard JM, Smith GP, Safory H, Neame S, Kaplan E, Rosenberg D, Zubedat S, Foltyn VN, Christoffersen CT, Bundgaard C, Thomsen C, Avital A, Christensen KV, Wolosker H (2017) Asc-1 transporter regulation of synaptic activity via the tonic release of $\mathrm{D}$-serine in the forebrain. Cereb Cortex 27:1573-1587.

Schell MJ, Molliver ME, Snyder SH (1995) D-serine, an endogenous synaptic modulator: localization to astrocytes and glutamate-stimulated release. Proc Natl Acad Sci U S A 92:3948-3952.

Seyer P, Vandermoere F, Cassier E, Bockaert J, Marin P (2016) Physical and functional interactions between the serotonin transporter and the neutral amino acid transporter ASCT2. Biochem J 473:1953-1965.

Solbu TT, Bjørkmo M, Berghuis P, Harkany T, Chaudhry FA (2010) SAT1, a glutamine transporter, is preferentially expressed in GABAergic neurons. Front Neuroanat 4:1.

Strísovský K, Jirásková J, Mikulová A, Rulísek L, Konvalinka J (2005) Dual substrate and reaction specificity in mouse serine racemase: identification of high-affinity dicarboxylate substrate and inhibitors and analysis of the beta-eliminase activity. Biochemistry 44:13091-13100.

Sugawara M, Nakanishi T, Fei YJ, Huang W, Ganapathy ME, Leibach FH, Ganapathy V (2000) Cloning of an amino acid transporter with functional characteristics and tissue expression pattern identical to that of system A. J Biol Chem 275:16473-16477.

Tani H, Dulla CG, Huguenard JR, Reimer RJ (2010) Glutamine is required for persistent epileptiform activity in the disinhibited neocortical brain slice. J Neurosci 30:1288-1300.

Tofteng F, Hauerberg J, Hansen BA, Pedersen CB, Jørgensen L, Larsen FS (2006) Persistent arterial hyperammonemia increases the concentration of glutamine and alanine in the brain and correlates with intracranial pressure in patients with fulminant hepatic failure. J Cereb Blood Flow Metab 26:21-27.
Utsunomiya-Tate N, Endou H, Kanai Y (1996) Cloning and functional characterization of a system ASC-like $\mathrm{Na}+$-dependent neutral amino acid transporter. J Biol Chem 271:14883-14890.

Uwechue NM, Marx MC, Chevy Q, Billups B (2012) Activation of glutamate transport evokes rapid glutamine release from perisynaptic astrocytes. J Physiol 590:2317-2331.

Varoqui H, Zhu H, Yao D, Ming H, Erickson JD (2000) Cloning and functional identification of a neuronal glutamine transporter. J Biol Chem 275:4049-4054

Watson CM, Trainor PA, Radziewic T, Pelka GJ, Zhou SX, Parameswaran M, Quinlan GA, Gordon M, Sturm K, Tam PP (2008) Application of lacZ transgenic mice to cell lineage studies. Methods Mol Biol 461:149164.

Wolosker H, Radzishevsky I (2013) The serine shuttle between glia and neurons: implications for neurotransmission and neurodegeneration. Biochem Soc Trans 41:1546-1550.

Wolosker H, Blackshaw S, Snyder SH (1999) Serine racemase: a glial enzyme synthesizing D-serine to regulate glutamate-N-methyl-D-aspartate neurotransmission. Proc Natl Acad Sci U S A 96:13409-13414.

Wolosker H, Balu DT, Coyle JT (2016) The rise and fall of the D-serinemediated gliotransmission hypothesis. Trends Neurosci 39:712-721.

Wreden CC, Johnson J, Tran C, Seal RP, Copenhagen DR, Reimer RJ, Edwards RH (2003) The $\mathrm{H}^{+}$-coupled electrogenic lysosomal amino acid transporter LYAAT1 localizes to the axon and plasma membrane of hippocampal neurons. J Neurosci 23:1265-1275.

Yamada D, Kawabe K, Tosa I, Tsukamoto S, Nakazato R, Kou M, Fujikawa K, Nakamura S, Ono M, Oohashi T, Kaneko M, Go S, Hinoi E, Yoneda Y, Takarada T (2019) Inhibition of the glutamine transporter SNAT1 confers neuroprotection in mice by modulating the mTOR-autophagy system. Commun Biol 2:346.

Yao D, Mackenzie B, Ming H, Varoqui H, Zhu H, Hediger MA, Erickson JD (2000) A novel system A isoform mediating $\mathrm{Na}+$ /neutral amino acid cotransport. J Biol Chem 275:22790-22797. 